\title{
A MULTIBAND SEMICLASSICAL MODEL FOR SURFACE HOPPING QUANTUM DYNAMICS*
}

\author{
LIHUI $\mathrm{CHAI}^{\dagger}$, SHI JIN ${ }^{\ddagger}, \mathrm{QIN}^{\S} \mathrm{LI}^{\S}$, AND OMAR MORANDI ${ }^{\oplus}$
}

\begin{abstract}
In the paper we derive a semiclassical model for surface hopping allowing quantum dynamical nonadiabatic transition between different potential energy surfaces in which cases the classical Born-Oppenheimer approximation breaks down. The model is derived using the Wigner transform and Weyl quantization, and the central idea is to evolve the entire Wigner matrix rather than just the diagonal entries as was done previously in the adiabatic case. The off-diagonal entries of the Wigner matrix suitably describe the nonadiabatic transition, such as the Berry connection, for avoided crossings. We study the numerical approximation issues of the model, and then conduct numerical experiments to validate the model.
\end{abstract}

Key words. semiclassical model, nonadiabatic transition, Wigner matrix, avoided crossings

AMS subject classifications. 81Q20, 81S30

DOI. $10.1137 / 140967842$

1. Introduction. In this paper we derive a semiclassical model based on the quantum phase-space description of the particle dynamics. We consider the nucleonic Schrödinger system:

$$
\begin{aligned}
\mathrm{i} \varepsilon \frac{\partial \psi^{\varepsilon}}{\partial t}(t, \boldsymbol{x}) & =\hat{H} \psi^{\varepsilon}(t, x), \quad(t, \boldsymbol{x}) \in\left(\mathbb{R}^{+}, \mathbb{R}^{d}\right), \\
\psi^{\varepsilon}(0, \boldsymbol{x}) & =\psi_{0}^{\varepsilon}(\boldsymbol{x})
\end{aligned}
$$

with the self-adjoint Hamiltonian operator defined by

$$
\hat{H}=-\frac{\varepsilon^{2}}{2} \Delta_{\boldsymbol{x}}+\tilde{V}(\boldsymbol{x})
$$

Here, $\psi$ is a vector and $\tilde{V}(x)$ is a Hermitian matrix. $\varepsilon=\sqrt{\frac{m}{M}}$ is the mass ratio between electron and nuclei. This system arises from the Born-Oppenheimer approximation [5] of the $N$-body Schrödinger equation in which the nucleonic Schrödinger system (1.1) is solved along the electronic potential surfaces. We will focus on the two-energy system although our study can be extended to systems with more energy levels in a

${ }^{*}$ Received by the editors May 5, 2014; accepted for publication (in revised form) October 27, 2014; published electronically January 20, 2015. This work was partially supported by NSF grants DMS-1114546 and DMS-1107291: NSF Research Network in Mathematical Sciences KI-Net: Kinetic Description of Emerging Challenges in Multiscale Problems of Natural Sciences.

http://www.siam.org/journals/mms/13-1/96784.html

$\dagger$ Department of Mathematics, University of California, Santa Barbara, Santa Barbara, CA 93106 (chailh@math.ucsb.edu).

¥Department of Mathematics, Institute of Natural Sciences and MOE-LSE, Shanghai Jiao Tong University, Shanghai 200240, China, and Department of Mathematics, University of WisconsinMadison, Madison, WI 53706 (jin@math.wisc.edu).

$\S$ Department of Computing and Mathematical Sciences, California Institute of Technology, Pasadena, CA 91125 (qinli@caltech.edu). This author's research was supported in part by the AFOSR MURI grant FA9550-09-1-0613 and the National Science Foundation under award DMS1318377.

I Institut de Physique et Chimie des Matériaux de Strasbourg, CNRS and University of Strasbourg, F-67034 Strasbourg, France (omar.morandi@ipcms.unistra.fr). 
straightforward way. In the two-energy level case, the potential matrix reads as

$$
\tilde{V}(\boldsymbol{x})=\frac{1}{2} \operatorname{tr} \tilde{V}(\boldsymbol{x})+V=U(\boldsymbol{x})+\left(\begin{array}{cc}
u(\boldsymbol{x}) & v(\boldsymbol{x}) \\
v^{\dagger}(\boldsymbol{x}) & -u(\boldsymbol{x})
\end{array}\right) .
$$

For future reference, we consider the unitary matrix $\Theta$,

$$
\Theta^{\dagger}(\boldsymbol{x})=\left[\chi_{+}, \chi_{-}\right]
$$

that diagonalizes the potential operator $V$. We have

$$
V=\Theta^{\dagger} \Lambda_{V} \Theta
$$

where

$$
\begin{aligned}
\Lambda_{V}(\boldsymbol{x}) & =\operatorname{diag}(E(\boldsymbol{x}),-E(\boldsymbol{x})) \\
& =\operatorname{diag}\left(\sqrt{|u(\boldsymbol{x})|^{2}+|v(\boldsymbol{x})|^{2}},-\sqrt{|u(\boldsymbol{x})|^{2}+|v(\boldsymbol{x})|^{2}}\right)
\end{aligned}
$$

and

$$
\Theta=\frac{1}{\sqrt{2\left(1+\frac{u(\boldsymbol{x})}{E(\boldsymbol{x})}\right)}}\left(\begin{array}{rr}
\left(1+\frac{u(\boldsymbol{x})}{E(\boldsymbol{x})}\right) \frac{v^{\dagger}(\boldsymbol{x})}{|v(\boldsymbol{x})|} & \frac{|v(\boldsymbol{x})|}{E(\boldsymbol{x})} \\
-\frac{v^{\dagger}(\boldsymbol{x})}{E(\boldsymbol{x})} & 1+\frac{u(\boldsymbol{x})}{E(\boldsymbol{x})}
\end{array}\right) .
$$

Obviously, $\chi_{ \pm}$are the eigenvectors of $V$ corresponding to the eigenvalues $\pm E$ with $E(\boldsymbol{x})=\sqrt{|u(\boldsymbol{x})|^{2}+|v(\boldsymbol{x})|^{2}}$. Hereafter, we call the two eigenvalues the energy bands, and $\Delta E=2 E$, the energy gap.

For $\Delta E=0$, the matrix $\Theta$ becomes singular (conical crossing). In this paper, we are interested in the cases where the energy gap is strictly positive and asymptotically small. In particular, we focus on the so-called avoided crossing scaling where the minimum of the energy gap is of the order $\sqrt{\varepsilon}$.

We consider a few types of prototype potentials and analyze their influence on the nonadiabatic transitions process:

One-dimensional $(1 D)$ case:

$$
u(x)=x, \quad v(x, \delta) \equiv \delta, \quad U(\boldsymbol{x})=0 .
$$

The eigenvalues are $\Lambda_{V}^{ \pm}= \pm E= \pm \sqrt{x^{2}+\delta^{2}}$, and the avoided crossing point is $x=0$.

Two-dimensional $(2 D)$ cases: The first example is

$$
u(\boldsymbol{x}, \delta)=x, \quad v(\boldsymbol{x}, \delta)=\sqrt{y^{2}+\delta^{2}}, \quad U(\boldsymbol{x})=0 .
$$

The second example is

$$
u(\boldsymbol{x}, \delta)=x, \quad v(\boldsymbol{x}, \delta)=y+\mathrm{i} \delta, \quad U(\boldsymbol{x})=0 .
$$

Here, we denote $\boldsymbol{x}=(x, y)$. In the $2 \mathrm{D}$ cases, the eigenvalues are given by $\Lambda_{V}^{ \pm}= \pm E= \pm \sqrt{x^{2}+y^{2}+\delta^{2}}$, and the avoided crossing point is $(x, y)=$ $(0,0)$.

Copyright $@$ by SIAM. Unauthorized reproduction of this article is prohibited. 
We are interested in deriving a semiclassical approximation to the Schrödinger system (1.1) with avoided-crossings. One of the advantages of our method is that the computational cost is significantly reduced compared to directly solving the original Schrödinger system (1.1).

One of the difficulties in the derivation of the semiclassical expansion for a system with two or more energy levels is the noncommutativity of the matrix $\tilde{V}$ with the Laplacian operator. In the case where $\Delta E=O(1)$, the equation of motion can be well approximated by a fully diagonalized system - one classical Liouville equation for each energy level $[30,27,12,13,14,19,1,38,41,37]$. This is the standard BornOppenheimer approximation. See reviews [20,42]. However, when the eigenvalues are of $O(\sqrt{\varepsilon})$ away from each other, the classical Born-Oppenheimer approximation breaks down and the diagonalized system is no longer a good approximation of the full coupled system. In such a case, around the crossing points, the particles could move from one band to another (the nonadiabatic phenomenon).

The study of the mathematical properties and the study of the physical systems where the energy band structure shows some crossing points date back to Wigner and von Neumann [45]. It can be shown that the crossing set is of measure zero, while the influence is of order 1 , and it is this crossing phenomenon that is responsible for some chemical reactions $[47,49]$. Due to its physical significance, this topic has been studied extensively in the computational chemistry community. The first result on the transition rate is due to Landau and Zener [50], who gave a rough estimate on the transition probability. Afterwards, there is very rich literature investigating the different aspects of the problem, including theoretical studies and algorithm development. We mention here the two most well-known algorithms, both by Tully et al.: the surface hopping method based on applying the Landau-Zener formula [43], and the fewest switches method [44], a Markov-chain Monte-Carlo-type method. Some criticisms have also been raised on the Landau-Zener formula, and we mention [3, 21].

On the mathematical side, in [15] Hagedorn first rigorously reexamined Zener's idea. This was followed by a series of works $[28,27,23,18,17,40,6]$, in which the authors also show that the jumping behavior could heavily depend on the types of crossings (see classification of crossings in [16]). The study of the nonadiabatic transition on the phase-space was done in [8].

The surface hopping algorithms that use the Landau-Zener formula for evaluating the nonadiabatic transitions for conical crossings have seen recent mathematical interest $[31,25,24,9]$. The main advantage of these surface hopping methods, compared to computing the original Schrödinger equation (1.1), is that they do not need to numerically resolve the $O(\varepsilon)$ wavelength. However, these methods cannot account for phase information at the crossing points, and thus ignore important physical phenomena $[4,35]$. The main result of this paper is to present a semiclassical model that includes the particle phase correction at the crossing points.

Our method is based on the Wigner transform [39] and the Weyl quantization $[46,22]$ procedure. In the adiabatic case, with $\varepsilon \rightarrow 0$ (classical limit), the Wigner transformation leads to a set of decoupled Liouville equations, each for one energy band $[39,10]$. In this case, only the diagonal entries of the Wigner matrix that correspond to the projection onto the two eigenspaces of the underlying Hamiltonian are relevant. However, in the presence of a band crossing, one cannot ignore the off-diagonal terms. For this reason, our main idea in the paper is to find the semiclassical approximation for the entire Wigner matrix. This approach is similar to the derivation of the transport equation for graphene [36] and in semiconductor systems 
[33]. Our model is a coupled Liouville system for all entries of the Wigner matrix, where the off-diagonal terms prescribe the quantum transition between bands and the two-band correlations due to the Berry connections. We also discuss numerical approximation of this model utilizing a multiphysics domain decomposition idea proposed in [7]: away from the crossing points we solve the standard adiabatic Liouville equations, while in the crossing zones the new semiclassical system is solved, and the two systems are connected by interface conditions.

In section 2 we present the derivation of the new semiclassical model. We also produce a primitive analysis of the behavior of the solutions to the system. In section 3 we describe a coupling method that combines the new semiclassical model near the crossing points with the adiabatic system elsewhere in order to further reduce the computational cost. Numerical examples are shown afterwards.

2. The semiclassical formulation. In the following we describe the basics of the Wigner transform and the Weyl quantization. In subsections 2.2 and 2.3 we derive the mathematical model for the adiabatic and nonadiabatic cases, respectively.

2.1. The Wigner transformation and Weyl quantization. The Wigner function is defined by

$$
F^{\varepsilon}(\boldsymbol{x}, \boldsymbol{p})=\frac{1}{(2 \pi)^{d}} \int \rho^{\varepsilon}\left(\boldsymbol{x}-\frac{\varepsilon \boldsymbol{y}}{2}, \boldsymbol{x}+\frac{\varepsilon \boldsymbol{y}}{2}\right) \mathrm{e}^{\mathrm{i} \boldsymbol{p} \cdot \boldsymbol{y}} \mathrm{d} \boldsymbol{y},
$$

where $\rho^{\varepsilon}\left(\boldsymbol{x}, \boldsymbol{x}^{\prime}\right)=\psi^{\varepsilon}(\boldsymbol{x}) \otimes \overline{\psi^{\varepsilon}}\left(\boldsymbol{x}^{\prime}\right)$ is the density matrix, $\psi^{\varepsilon}$ is defined in (1.1), $\overline{\psi^{\varepsilon}}$ is the complex conjugate of $\psi^{\varepsilon}$. The Wigner function is defined in a quadratic manner, so it is insensitive to a constant phase shift.

The moments of the Wigner distribution function taken with respect to the momentum variable provide the physical observables of the system. In particular, the position density and flux are given by

$$
\rho^{\varepsilon}(\boldsymbol{x}, t)=\left|\psi^{\varepsilon}\right|^{2}=\int_{\mathbb{R}^{d}} F^{\varepsilon} \mathrm{d} \boldsymbol{p}, \quad J^{\varepsilon}(\boldsymbol{x}, t)=\varepsilon \operatorname{Im}\left(\bar{\psi}^{\varepsilon} \cdot \nabla_{\boldsymbol{x}} \psi^{\varepsilon}\right)=\int_{\mathbb{R}^{d}} \boldsymbol{p} F^{\varepsilon} \mathrm{d} \boldsymbol{p} .
$$

The evolution of the Wigner function is governed by the Wigner equation

$$
\partial_{t} F^{\varepsilon}+\boldsymbol{p} \cdot \nabla_{\boldsymbol{x}} F^{\varepsilon}+\Xi[U \mathbb{I}+V] F^{\varepsilon}=0,
$$

where $\Xi[V]$ is defined as

$$
\begin{aligned}
\Xi[V] F^{\varepsilon}=\frac{\mathrm{i}}{\varepsilon(2 \pi)^{d}} \int_{\mathbb{R}^{2 d}} \mathrm{~d} \boldsymbol{p}^{\prime} \mathrm{d} \boldsymbol{y} \mathrm{e}^{\mathrm{i}\left(\boldsymbol{p}^{\prime}-\boldsymbol{p}\right) \cdot \boldsymbol{y}} \\
\times\left[V\left(\boldsymbol{x}-\frac{\varepsilon \boldsymbol{y}}{2}\right) F^{\varepsilon}\left(\boldsymbol{x}, \boldsymbol{p}^{\prime}\right)-F^{\varepsilon}\left(\boldsymbol{x}, \boldsymbol{p}^{\prime}\right) V\left(\boldsymbol{x}+\frac{\varepsilon \boldsymbol{y}}{2}\right)\right] .
\end{aligned}
$$

We note that $F$ and $V$ are matrices, and in general they do not commute.

A quantum mechanical operator can be univocally associated to a function $A(\boldsymbol{x}, \boldsymbol{p})$ defined on the classical phase-space by the so-called Weyl quantization [46, 34]. The following map is used:

$$
\mathcal{W}(A)[h](\boldsymbol{x})=\hat{A}[h](\boldsymbol{x})=\frac{1}{(2 \pi \varepsilon)^{d}} \iint A\left(\frac{\boldsymbol{x}+\boldsymbol{y}}{2}, \boldsymbol{p}\right) h(\boldsymbol{x}, \boldsymbol{y}) \mathrm{e}^{\frac{\mathrm{i}}{\varepsilon}(\boldsymbol{x}-\boldsymbol{y}) \cdot \boldsymbol{p}} \mathrm{d} \boldsymbol{p} \mathrm{d} \boldsymbol{y} .
$$

Here, $\hat{A} \equiv \mathcal{W}(A)$ is the Weyl quantum mechanical operator defined on the space of the smooth functions $h(\boldsymbol{x}, \boldsymbol{y}) \in \mathcal{S}\left(\mathbb{R}^{d} \times \mathbb{R}^{d}\right)$. The function $A(\boldsymbol{x}, \boldsymbol{p})$ denotes the 
symbol of $\hat{A}$. It is easy to verify that the Weyl quantization map is the inverse of the Wigner transform (the Weyl quantization procedure applied to the Wigner function $F^{\varepsilon}$ provides the density operator).

In particular, the Weyl quantization of the Hamiltonian is the Schrödinger operator. Namely,

$$
A(\boldsymbol{x}, \boldsymbol{p})=H(\boldsymbol{x}, \boldsymbol{p})=\frac{\boldsymbol{p}^{2}}{2}+\tilde{V}(\boldsymbol{x}) \quad \Rightarrow \quad \hat{A}=\hat{H}=-\frac{\varepsilon^{2}}{2} \Delta_{\boldsymbol{x}}+\tilde{V}(\boldsymbol{x}) .
$$

The use of the Wigner-Moyal formalism is eased by the definition of the Moyal product $\#$ as

$$
\begin{aligned}
A \# B= & \frac{1}{(2 \pi)^{2 d}} \int A\left(\boldsymbol{x}-\frac{\varepsilon}{2} \boldsymbol{\eta}, \boldsymbol{p}+\frac{\varepsilon}{2} \boldsymbol{\mu}\right) B\left(\boldsymbol{x}^{\prime}, \boldsymbol{p}^{\prime}\right) \\
& \times \mathrm{e}^{\mathrm{i}\left(\boldsymbol{x}-\boldsymbol{x}^{\prime}\right) \cdot \boldsymbol{\mu}+\mathrm{i}\left(\boldsymbol{p}-\boldsymbol{p}^{\prime}\right) \cdot \boldsymbol{\eta}} \mathrm{d} \boldsymbol{\mu} \mathrm{d} \boldsymbol{x}^{\prime} \mathrm{d} \boldsymbol{\eta} \mathrm{d} \boldsymbol{p}^{\prime} \\
= & A \mathrm{e}^{\frac{\mathrm{i} \varepsilon}{2}\left(\overleftarrow{\nabla}_{\boldsymbol{x}} \cdot \vec{\nabla}_{\boldsymbol{p}}-\overleftarrow{\nabla}_{\boldsymbol{p}} \cdot \vec{\nabla}_{\boldsymbol{x}}\right)} B,
\end{aligned}
$$

where the arrows indicate on which symbol the gradients act. An important property of the Moyal product is $\mathcal{W}(A \# B)=\mathcal{W}(A) \mathcal{W}(B)$. The \#-product admits an $\varepsilon$ expansion. The $O(\varepsilon)$ term is the classical Poisson bracket $\{A, B\}=\nabla_{\boldsymbol{p}} A \cdot \nabla_{\boldsymbol{x}} B-$ $\nabla_{\boldsymbol{x}} A \cdot \nabla_{\boldsymbol{p}} B$, and

$$
A \# B=A B-\frac{\mathrm{i} \varepsilon}{2}\{A, B\}+O\left(\varepsilon^{2}\right) .
$$

2.2. The adiabatic case. The mathematical study of the semiclassical limit in the adaibatic case was carried out in [39, 10]. According to Theorem 6.1 in [10], outside the crossing set $S=\left\{\boldsymbol{x}: \lambda^{+}(\boldsymbol{x})=\lambda^{-}(\boldsymbol{x})\right\}$, the Wigner function can be obtained by the projection of the solution onto the eigenspaces of the Hamiltonian. Let $F^{0}(t, \boldsymbol{x}, \boldsymbol{p}) \doteq \lim _{\varepsilon \rightarrow 0} F(t, \boldsymbol{x}, \boldsymbol{p})$; we have

$$
\begin{aligned}
F^{0}(t, \cdot) & =\Pi_{+} F^{0}(t, \cdot) \Pi_{+}+\Pi_{-} F^{0}(t, \cdot) \Pi_{-} \\
& =f^{+}(t, \cdot) \Pi_{+}+f^{-}(t, \cdot) \Pi_{-},
\end{aligned}
$$

where

$$
\Pi_{ \pm}(\boldsymbol{x})=\chi^{ \pm}(\boldsymbol{x}) \otimes \chi^{ \pm}(\boldsymbol{x})
$$

and $f^{ \pm}$are the particle densities related to energy levels $\lambda^{ \pm}(\boldsymbol{x}, \boldsymbol{k})$ given by

$$
f^{ \pm}=\operatorname{Tr}\left(\Pi_{ \pm} F^{0}(t, \cdot)\right)
$$

The distributions $f^{ \pm}$satisfy the classical Liouville equation:

$$
\begin{aligned}
\partial_{t} f^{ \pm}+\nabla_{\boldsymbol{p}} \lambda^{ \pm} \cdot \nabla_{\boldsymbol{x}} f^{ \pm}-\nabla_{\boldsymbol{x}} \lambda^{ \pm} \cdot \nabla_{\boldsymbol{p}} f^{ \pm} & =0, \quad t>0, \boldsymbol{x} \in \mathbb{R}^{d} \backslash S, \boldsymbol{p} \in \mathbb{R}^{d}, \\
f^{ \pm}(t=0, \boldsymbol{x}, \boldsymbol{p}) & =\operatorname{Tr}\left(\Pi_{ \pm} F^{0}(t=0, \boldsymbol{x}, \boldsymbol{p})\right) .
\end{aligned}
$$

2.3. Quantum transition in the nonadiabatic case. In the proximity of the crossing points, the Born-Oppenheimer approximation is no longer valid. By using the Wigner formalism, we derive a semiclassical model that is able to treat the quantum mechanical band transitions in the case where the separation between the 
upper and lower energy levels scales as $\sqrt{\varepsilon}$. In particular, the transitions between bands are captured by the off-diagonal terms of the Wigner matrix distribution. Our approach is the alternative to the use of the Landau-Zener formula for the evaluation of the transition probability in correspondence to an avoided-crossing and overcomes some of the difficulties that affect the Landau-Zener approach as argued in [21]. We follow the derivation presented in [36, 29].

Starting from the von Neumann equation, we have

$$
\mathrm{i} \varepsilon \frac{\partial \hat{F}^{\prime}}{\partial t}=\left[\hat{H}^{\prime}, \hat{F}^{\prime}\right]
$$

where we have defined

$$
\hat{F}^{\prime}=\hat{\Theta} \hat{\rho} \hat{\Theta}^{\dagger}, \quad \hat{H}^{\prime}=\hat{\Theta} \hat{H} \hat{\Theta}^{\dagger} .
$$

Here, $\hat{\Theta}=\mathcal{W}[\Theta]$ and $\hat{\Theta}^{\dagger}$ are the Weyl quantization of $\Theta$ and $\Theta^{\dagger}$, respectively. In particular, $\hat{\Theta} \hat{V} \hat{\Theta}^{\dagger}=\hat{\Lambda}_{V}$.

Equation (2.11) is a diagonalized version of the von Neumann equation written on the Weyl operator formalism. To obtain an equivalent dynamical system defined on the quantum phase-space, we used the inverse Weyl mapping:

$$
\mathrm{i} \varepsilon \frac{\partial F^{\prime}}{\partial t}=\left[H^{\prime}, F^{\prime}\right]_{\#},
$$

where $[A, B]_{\#}=A \# B-B \# A$ is a commutator of the Moyal product (2.6), and $H^{\prime}$ and $F^{\prime}$ are symbols associated with $\hat{H}^{\prime}$ and $\hat{F}^{\prime}$, respectively:

$$
H^{\prime}(\boldsymbol{x}, \boldsymbol{p})=\Theta(\boldsymbol{x}) \# H(\boldsymbol{x}, \boldsymbol{p}) \# \Theta(\boldsymbol{x})^{\dagger}, \quad F^{\prime}(\boldsymbol{x}, \boldsymbol{p})=\Theta(\boldsymbol{x}) \# F(\boldsymbol{x}, \boldsymbol{p}) \# \Theta(\boldsymbol{x})^{\dagger} .
$$

Further, we get (see the appendix for details)

$$
H^{\prime}(\boldsymbol{x}, \boldsymbol{p})=\Lambda(\boldsymbol{x}, \boldsymbol{p})+\mathrm{i} \varepsilon \boldsymbol{p} \cdot \nabla_{\boldsymbol{x}} \Theta(\boldsymbol{x}) \Theta^{\dagger}(\boldsymbol{x})+\frac{\varepsilon^{2}}{2} \nabla_{\boldsymbol{x}} \Theta(\boldsymbol{x}) \cdot \nabla_{\boldsymbol{x}} \Theta^{\dagger}(\boldsymbol{x}) .
$$

By using (2.7), we expand (2.13) and get

$$
\begin{aligned}
\frac{\partial F^{\prime}}{\partial t} & =\frac{1}{\mathrm{i} \varepsilon}\left[\Lambda, F^{\prime}\right]-\frac{1}{2}\left\{\Lambda, F^{\prime}\right\}+\frac{1}{2}\left\{F^{\prime}, \Lambda\right\}+\left[\boldsymbol{p} \cdot \nabla_{\boldsymbol{x}} \Theta \Theta^{\dagger}, F^{\prime}\right]+R^{\varepsilon} \\
(2.16) & =\frac{1}{\mathrm{i} \varepsilon}\left[\Lambda, F^{\prime}\right]-\frac{1}{2}\left[\nabla_{\boldsymbol{p}} \Lambda, \nabla_{\boldsymbol{x}} F^{\prime}\right]_{+}+\frac{1}{2}\left[\nabla_{\boldsymbol{x}} \Lambda, \nabla_{\boldsymbol{p}} F^{\prime}\right]_{+}+\left[\boldsymbol{p} \cdot \nabla_{\boldsymbol{x}} \Theta \Theta^{\dagger}, F^{\prime}\right]+R^{\varepsilon},
\end{aligned}
$$

with $[A, B]_{+}=A B+B A$. Here $R^{\varepsilon}$ is the remainder term, and if one formally expands out the Moyal product, the leading order terms in $R^{\varepsilon}$ are

$$
-\frac{\mathrm{i} \varepsilon}{2}\left[\nabla_{\boldsymbol{x}} \Theta \cdot \nabla_{\boldsymbol{x}} \Theta^{\dagger}, F^{\prime}\right]-\frac{\mathrm{i} \varepsilon}{2}\left\{\boldsymbol{p} \cdot \nabla_{\boldsymbol{x}} \Theta \Theta^{\dagger}, F^{\prime}\right\}+\frac{\mathrm{i} \varepsilon}{2}\left\{F^{\prime}, \boldsymbol{p} \cdot \nabla_{\boldsymbol{x}} \Theta \Theta^{\dagger}\right\} .
$$

Formally, the $R^{\varepsilon}$ term in the above equation is of $O(\varepsilon)$ and is negligible. We throw it away for the evolution equations below. Details on the asymptotic derivation are found in the appendix. We then have the following:

$$
\begin{aligned}
& \frac{\partial f^{+}}{\partial t}=-\boldsymbol{p} \cdot \nabla_{\boldsymbol{x}} f^{+}+\nabla_{\boldsymbol{x}}(U+E) \cdot \nabla_{\boldsymbol{p}} f^{+}+\bar{b}^{i} f^{i}+b^{i} \overline{f^{i}} \\
& \frac{\partial f^{-}}{\partial t}=-\boldsymbol{p} \cdot \nabla_{\boldsymbol{x}} f^{-}+\nabla_{\boldsymbol{x}}(U-E) \cdot \nabla_{\boldsymbol{p}} f^{-}-\bar{b}^{i} f^{i}-b^{i} \overline{f^{i}} \\
& \frac{\partial f^{i}}{\partial t}=-\boldsymbol{p} \cdot \nabla_{\boldsymbol{x}} f^{i}+\nabla_{\boldsymbol{x}} U \cdot \nabla_{\boldsymbol{p}} f^{i}+b^{i}\left(f^{-}-f^{+}\right)+\left(b^{+}-b^{-}\right) f^{i}+\frac{2 E}{\mathrm{i} \varepsilon} f^{i}
\end{aligned}
$$

Copyright (c) by SIAM. Unauthorized reproduction of this article is prohibited. 
where we have denoted

$$
F^{\prime}=\left(\begin{array}{cc}
f^{+} & f^{i} \\
\bar{f}^{i} & f^{-}
\end{array}\right) \text {and } \boldsymbol{p} \cdot \nabla_{\boldsymbol{x}} \Theta \Theta^{\dagger}=\left(\begin{array}{cc}
b^{+} & b^{i} \\
-\bar{b}^{i} & b^{-}
\end{array}\right) .
$$

In vector form, (2.18) becomes

$$
\frac{\partial \boldsymbol{f}}{\partial t}+\boldsymbol{p} \cdot \nabla_{\boldsymbol{x}} \boldsymbol{f}-\nabla_{\boldsymbol{x}} A \cdot \nabla_{\boldsymbol{p}} \boldsymbol{f}=C \boldsymbol{f}+\frac{D}{\mathrm{i} \varepsilon} \boldsymbol{f},
$$

where

$$
\begin{aligned}
\boldsymbol{f} & =\left(f^{+}, f^{-}, f^{i}, \overline{f^{i}}\right)^{T}, \\
A & =\operatorname{diag}(U+E, U-E, U, U), \\
D & =\operatorname{diag}(0,0,2 E,-2 E), \\
C & =\left(\begin{array}{cccc}
0 & 0 & \overline{b^{i}} & b^{i} \\
0 & 0 & -\overline{b^{i}} & -b^{i} \\
-b^{i} & b^{i} & b^{+}-b^{-} & 0 \\
-\overline{b^{i}} & \overline{b^{i}} & 0 & b^{+}-b^{-}
\end{array}\right) .
\end{aligned}
$$

Here, $f^{ \pm}$, both real, represent the projection coefficients onto the positive and negative energy bands. The function $f^{i}$ describes the transition between the two bands.

Specifically for the three examples in (1.9), (1.10) and (1.11), we have explicit formulae for $b^{s}, s \in\{ \pm, i\}$ :

For (1.9): $b^{+}=b^{-} \equiv 0, \quad$ and $\quad b^{i}=-\frac{p \delta}{2\left(x^{2}+\delta^{2}\right)}$.

For (1.10): denote $\boldsymbol{p}=(p, q)$, then

$$
b^{+}=b^{-} \equiv 0, \quad b^{i}=\frac{1}{2\left(x^{2}+y^{2}+\delta^{2}\right)}\left(\frac{q x y}{\sqrt{y^{2}+\delta^{2}}}-p \sqrt{y^{2}+\delta^{2}}\right) .
$$

For (1.11): one has for $\boldsymbol{p}=(p, q), E=\sqrt{x^{2}+y^{2}+\delta^{2}}$ and

$$
\begin{aligned}
b^{+} & =\frac{q \delta(E+x)}{2 E^{3}} \mathrm{i}, \quad b^{-}=\frac{q \delta}{2 E(E+x)} \mathrm{i}, \\
b^{i} & =\frac{1}{2\left(x^{2}+y^{2}+\delta^{2}\right)}\left\{\left(\frac{q x y}{\sqrt{y^{2}+\delta^{2}}}-p \sqrt{y^{2}+\delta^{2}}\right)-\mathrm{i} q \delta\right\} .
\end{aligned}
$$

The system (2.20) is hyperbolic, $\Theta$ is unitary, $b^{ \pm}$are purely imaginary, and the matrix $C$ is skew Hermitian.

Remark 2.1. According to the adiabatic theory $[48,36]$, when time $t$ is sufficiently small, the solution of the Schrödinger equation (1.1) can be written as

$$
\begin{aligned}
\psi^{\varepsilon}(t) & =\psi_{+}(t)+\psi_{-}(t), \\
\text { with } \psi_{ \pm}(t) & =\mathrm{e}^{\mathrm{i} \gamma_{ \pm}(t)} \exp \left(\mp \frac{\mathrm{i}}{\varepsilon} \int_{0}^{t} \mathrm{~d} t^{\prime} E\left(\boldsymbol{x}\left(t^{\prime}\right)\right)\right) \chi_{ \pm}(\boldsymbol{x}(t)),
\end{aligned}
$$

where $\boldsymbol{x}(t)$ is a semiclassical trajectory. For $t=0$, the initial state coincides with the eigenstate $\chi_{s}(\boldsymbol{x}(0))$ for $s \in\{+,-\}$. The second exponential in (2.23) is known as 
the dynamical phase factor, and $\gamma_{ \pm}$in the first exponential is the path integral of the Berry connection, i.e.,

$$
\gamma_{ \pm}(t)=\mathrm{i} \int \dot{\boldsymbol{x}}(t) \cdot\left(\nabla_{\boldsymbol{x}} \chi_{ \pm}(\boldsymbol{x}(t)) \cdot \chi_{ \pm}^{\dagger}(\boldsymbol{x}(t))\right) \mathrm{d} t
$$

which is called the Berry phase. This term cancels out in the diagonal term of the density function $\psi_{+}^{\dagger} \psi_{+}$and $\psi_{-}^{\dagger} \psi_{-}$. However, for the off-diagonal term $\psi_{-}^{\dagger} \psi_{+}$, we have

$$
\psi_{-}^{\dagger} \psi_{+}(\boldsymbol{x}(t))=\exp \left\{\mathrm{i}\left(\gamma_{+}(t)-\gamma_{-}(t)-\frac{2}{\varepsilon} \int_{0}^{t} \mathrm{~d} t^{\prime} E\left(\boldsymbol{x}\left(t^{\prime}\right)\right)\right)\right\} \chi_{-}^{\dagger}(\boldsymbol{x}(t)) \chi_{+}(\boldsymbol{x}(t)) .
$$

By evaluating the derivative of the Berry phase, we have

$$
\begin{aligned}
& \mathrm{i} \frac{\mathrm{d}}{\mathrm{d} t}\left(\gamma_{+}(t)-\gamma_{-}(t)-\frac{2}{\varepsilon} \int_{0}^{t} \mathrm{~d} t^{\prime} E\left(\boldsymbol{x}\left(t^{\prime}\right)\right)\right) \\
= & -\dot{\boldsymbol{x}}(t) \cdot\left(\nabla_{\boldsymbol{x}} \chi_{+} \cdot \chi_{+}^{\dagger}-\nabla_{\boldsymbol{x}} \chi_{-} \cdot \chi_{-}^{\dagger}\right)-\frac{2 \mathrm{i}}{\varepsilon} E(\boldsymbol{x}(t)) .
\end{aligned}
$$

If we apply $\dot{\boldsymbol{x}}=\boldsymbol{p}$ where $\boldsymbol{p}$ is the momentum, then we get

$$
\begin{aligned}
& \mathrm{i} \frac{\mathrm{d}}{\mathrm{d} t}\left(\gamma_{+}(t)-\gamma_{-}(t)-\frac{2}{\varepsilon} \int_{0}^{t} \mathrm{~d} t^{\prime} E\left(\boldsymbol{x}\left(t^{\prime}\right)\right)\right) \\
= & \left(b^{+}(\boldsymbol{x}(t))-b^{-}(\boldsymbol{x}(t))\right)-\frac{2 \mathrm{i}}{\varepsilon} E(\boldsymbol{x}(t)) .
\end{aligned}
$$

Comparing with $(2.18 \mathrm{c})$, one can see that these are exactly the coefficients of the $f^{i}$ terms in (2.18c). This shows our model indeed captures the Berry phase in the interband transition processes.

3. A hybrid model by domain decomposition. Equation (2.18) is a hyperbolic system, with a transport part and a source term. Concerning the numerical treatment of (2.18), the major difficulties arise from the term $\frac{2 E}{\mathrm{i} \varepsilon}$ in the equation for $f^{i}$. It introduces rapid oscillations in both space and time that demand high computational cost. In order to reduce the numerical complexity, we solve the semiclassical model (2.18) only in the proximity of the crossing zone. Away from the crossing points we neglect the band transitions and solve the adiabatic model

$$
\begin{aligned}
& \frac{\partial f^{+}}{\partial t}=-\boldsymbol{p} \cdot \nabla_{\boldsymbol{x}} f^{+}+\nabla_{\boldsymbol{x}}(U+E) \cdot \nabla_{\boldsymbol{p}} f^{+}, \\
& \frac{\partial f^{-}}{\partial t}=-\boldsymbol{p} \cdot \nabla_{\boldsymbol{x}} f^{-}+\nabla_{\boldsymbol{x}}(U-E) \cdot \nabla_{\boldsymbol{p}} f^{-} .
\end{aligned}
$$

A similar hybrid model was used in [7] for the Schrödinger equation with a periodic lattice potential.

As an example, consider the $1 \mathrm{D}$ case with $p>0$ (so that in the $x$ space the wave packet moves from the left to the right). The other cases are treated similarly. We decompose the domain into the following two regions:

The adiabatic region. $x<-C_{0} \sqrt{\varepsilon}$ or $x>C_{0} \sqrt{\varepsilon}$ :

In this region, we use $o(1)$ coarse mesh, independent of $\varepsilon$ for the adiabatic Liouville system (3.1). $f^{i}$ is set to be zero. At $x=-C_{0} \sqrt{\varepsilon}$, no boundary condition is required, while at $x=C_{0} \sqrt{\varepsilon}$, we impose the inflow boundary condition that $f^{+}$and $f^{-}$are given by the solution inside the nonadiabatic region discussed below. 
The nonadiabatic region. $\left[-C_{0} \sqrt{\varepsilon}, C_{0} \sqrt{\varepsilon}\right]$ :

In this region we use $o(\sqrt{\varepsilon})$ mesh and compute the full system (2.18). The system is hyperbolic, so the boundary condition need only be specified in the incoming direction. The incoming boundary data for $f^{i}$ is set to be zero. Since the region size is of $O(\sqrt{\varepsilon})$, the total number of grid points along the $x$-direction remains independent from $\varepsilon$.

Remark 3.1. In theory, as we truncate the nonadiabatic region at $O(\sqrt{\varepsilon})$, the typical band gap $2 E$ in this region is of $O(\sqrt{\varepsilon})$; then the $\frac{2 E}{\mathrm{i} \varepsilon}$ term in $(2.18 \mathrm{c})$ is of $O\left(\frac{1}{\sqrt{\epsilon}}\right)$. So we use $o(\sqrt{\varepsilon})$ mesh grids along $x$ and $p$ inside the domain. This in total leads to $O(1)$ grid points along $x$ and $O\left(\frac{1}{\sqrt{\varepsilon}}\right)$ grid points along $p$. In the adiabatic region, as no $\varepsilon$ exists, the computational cost is $O(1)$. Therefore, in general on the whole phase-space plane we have $O\left(\frac{1}{\sqrt{\varepsilon}}\right)$ grid points. In comparison, the Schrödingier equation is computed only on the physical space, but the mesh size is restrictive: discretization along the $x$ domain is $\Delta x=o(\varepsilon)$ meaning that one needs to sample $O\left(\frac{1}{\varepsilon}\right)$ grid points. So our method is better than the original computation in the zero limit of $\varepsilon \ll 1$.

4. Numerical examples. In our numerical simulation, we use the hybrid model proposed in the previous section. For the transport operator of the Liouville systems (both adiabatic and nonadiabatic cases), we use the standard second order upwind total-variation-diminishing (TVD) scheme with van Leer slope limiter [32]. The reference solutions are obtained by the direct computation of the Schrödinger equation (1.1) with the time-splitting spectral method described in [2]. In the examples presented in the next sections, we use the following initial data:

$$
\psi^{\varepsilon}(t=0, \boldsymbol{x})=\psi_{0}^{\varepsilon}(\boldsymbol{x})=g_{0}^{\varepsilon}(\boldsymbol{x})\left(a^{+} \chi^{+}(\boldsymbol{x})+a^{-} \chi^{-}(\boldsymbol{x})\right)
$$

where $g_{0}^{\varepsilon}$ is the $\varepsilon$-scaled Gaussian packet:

$$
g_{0}^{\varepsilon}(\boldsymbol{x})=\left(\frac{A_{0}}{\pi}\right)^{d / 4} \exp \left\{-\frac{A_{0}}{2}\left|\boldsymbol{x}-\boldsymbol{x}_{\mathbf{0}}\right|^{2}+\frac{\mathrm{i}}{\varepsilon} \boldsymbol{p}_{\mathbf{0}} \cdot\left(\boldsymbol{x}-\boldsymbol{x}_{\mathbf{0}}\right)\right\}
$$

Here, $a^{ \pm}$are constants and $\chi^{ \pm}$the eigenvectors of the operator $\hat{V}$ (see (1.4)). By using the definition of the Wigner transform (2.1) and (2.14), we obtain the initial condition for $F^{\prime}$ :

$$
\begin{aligned}
& f^{+}(t=0, \boldsymbol{x}, \boldsymbol{p})=\left(a^{+}\right)^{2}\left(\frac{A_{0}}{\pi^{2} \varepsilon}\right)^{d / 2} \exp \left\{-A_{0}\left|\boldsymbol{x}-\boldsymbol{x}_{\mathbf{0}}\right|^{2}-\frac{1}{A_{0} \varepsilon^{2}}\left|\boldsymbol{p}-\boldsymbol{p}_{\mathbf{0}}\right|^{2}\right\} \\
& f^{-}(t=0, \boldsymbol{x}, \boldsymbol{p})=\left(a^{-}\right)^{2}\left(\frac{A_{0}}{\pi^{2} \varepsilon}\right)^{d / 2} \exp \left\{-A_{0}\left|\boldsymbol{x}-\boldsymbol{x}_{\mathbf{0}}\right|^{2}-\frac{1}{A_{0} \varepsilon^{2}}\left|\boldsymbol{p}-\boldsymbol{p}_{\mathbf{0}}\right|^{2}\right\} \\
& f^{i}(t=0, \boldsymbol{x}, \boldsymbol{p})=a^{+} a^{-}\left(\frac{A_{0}}{\pi^{2} \varepsilon}\right)^{d / 2} \exp \left\{-A_{0}\left|\boldsymbol{x}-\boldsymbol{x}_{\mathbf{0}}\right|^{2}-\frac{1}{A_{0} \varepsilon^{2}}\left|\boldsymbol{p}-\boldsymbol{p}_{\mathbf{0}}\right|^{2}\right\}
\end{aligned}
$$

where, according to (2.20b), we have expressed the initial data in terms of the components of the vector $\boldsymbol{f}$. In particular, we note that in the limit $\varepsilon \rightarrow 0, f^{+}$and $f^{-}$ become the classical Dirac measure $\delta\left(\boldsymbol{p}-\boldsymbol{p}_{0}\right)$. 
In our numerical experiments, the relevant physical observables are the particle density in the lower (-) and upper $(+)$ bands. In order to compare the solution of our new model with the original Schrödinger equation, it is convenient to consider the expression of the particle density in the two formulations:

$$
\left\{\begin{array}{l}
\rho_{\text {schr }}^{ \pm}(t, \boldsymbol{x})=\left|\Pi_{ \pm} \psi^{\varepsilon}(t, \boldsymbol{x})\right|^{2} \quad \text { and } \quad P_{\text {schr }}^{ \pm}=\int_{\mathbb{R}^{d}} \rho_{\text {schr }}^{ \pm}(t, \boldsymbol{x}) \mathrm{d} \boldsymbol{x} \\
\rho_{\text {liou }}^{ \pm}(t, \boldsymbol{x})=\int_{\mathbb{R}^{d}} f^{ \pm}(t, \boldsymbol{x}, \boldsymbol{k}) \mathrm{d} \boldsymbol{k} \text { and } \quad P_{\text {liou }}^{ \pm}=\int_{\mathbb{R}^{d}} \rho_{\text {liou }}^{ \pm}(t, \boldsymbol{x}) \mathrm{d} \boldsymbol{x} .
\end{array}\right.
$$

The total density is given by $[11,26]$

$$
M_{\text {schr }}=\int_{\Omega_{x}}\left(\rho_{\text {schr }}^{+}(\boldsymbol{y})+\rho_{\text {schr }}^{-}(\boldsymbol{y})\right) \mathrm{d} \boldsymbol{y}, \quad M_{\text {liou }}=\int_{\Omega_{x}}\left(\rho_{\text {liou }}^{+}(\boldsymbol{y})+\rho_{\text {liou }}^{-}(\boldsymbol{y})\right) \mathrm{d} \boldsymbol{y}
$$

where $\Omega_{\boldsymbol{x}}=\left\{\boldsymbol{y}=\left(y_{1}, \ldots, y_{d}\right) \in \mathbb{R}^{d}: y_{i} \leq x_{i}, i=1, \ldots, d\right\}$ for $\boldsymbol{x}=\left(x_{1}, \ldots, x_{d}\right) \in$ $\mathbb{R}^{d}, \Omega \subset \mathbb{R}^{d}$ is the computational domain, and $|\Omega|$ is the measure of $\Omega$. In order to estimate the accuracy of our method, we define the following $L^{1}$-norm of the error:

$$
\operatorname{Err}^{\varepsilon}=\frac{1}{|\Omega|} \int_{\Omega}\left|M_{\text {schr }}-M_{\text {liou }}\right| \mathrm{d} \boldsymbol{x} .
$$

4.1. Test 1: 1D system. In our first example, we consider a 1D system. We choose $u$ and $v$ as

$$
u(x)=x \quad \text { and } \quad v(x) \equiv \delta=\frac{\sqrt{\varepsilon}}{4}
$$

The minimum of the energy gap is $2 \delta(x=0)$. The initial data for the Schrödinger equation are given in (4.1)-(4.2) with $a^{+}=1, a^{-}=0, x_{0}=0.3125$, and $p_{0}=-1$ (pure state initial condition). For the Schrödinger equation we use a uniform grid for the space and time variables with, respectively, $\Delta x=\varepsilon / 32$ and $\Delta t=\varepsilon / 32$. For the semiclassical Liouville system (2.18), the phase-space $(x, p)$ domain is discretized with a uniform mesh with $\Delta x=\Delta p=2^{-9}$ in the adiabatic region $(x<-3 \sqrt{\varepsilon}$ or $x>3 \sqrt{\varepsilon}$ ), and $\Delta x=\Delta p=2^{-11}$ in the nonadiabatic region. The time step is chosen as $\Delta t=2^{-14}$.

The evolution of the particle wave packets can be easily understood. The trajectories of the wave packets' center-of-mass are illustrated in Figure 1. The Gaussian profile has a negative mean velocity and passes through the crossing point $x=0$ at around $t=0.25$. The Gaussian wave function splits into two parts. Around one half of the particles enter into the lower energy band, and the others stay in the upper band. The packet on the lower energy level is accelerated and leaves the simulation domain. The particles on the higher energy band are accelerated on the opposite direction, the momentum decreases, and the wave packet is reflected around $x=-1$. At $t=2.75$, the wave passes through the crossing point for the second time and undergoes another hopping process. In Figure 2 we show the evolution of $P^{+}$with respect to time. As the wave packet passes through the crossing point twice, the mass gets transferred to another energy band twice, generating two jumps in $P^{+}$. The numerical results given by the semiclassical model show good agreement with that of the Schrödingier equation. 


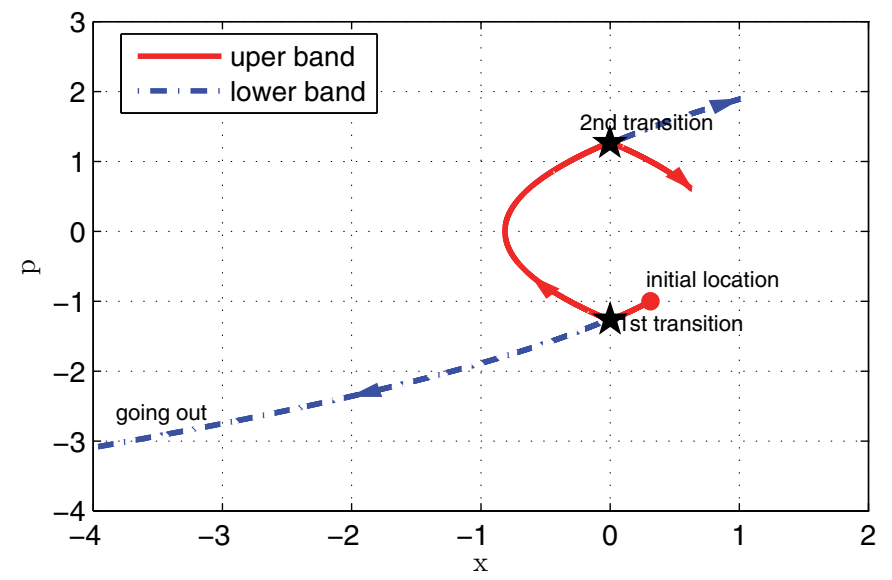

FIG. 1. Test 1: The trajectories of wave packets. Initially, the wave packet centers around the bullet point and starts moving towards the crossing point at $x=0$, marked as a star. It splits into two parts there: one of them, denoted by the dash-point line, keeps moving towards the left, while the other wave packet, the one that jumps up to the higher energy band, bounces back and hits the origin at $x=0$. Over there, it goes through the "second transition" and splits up into trajectories.

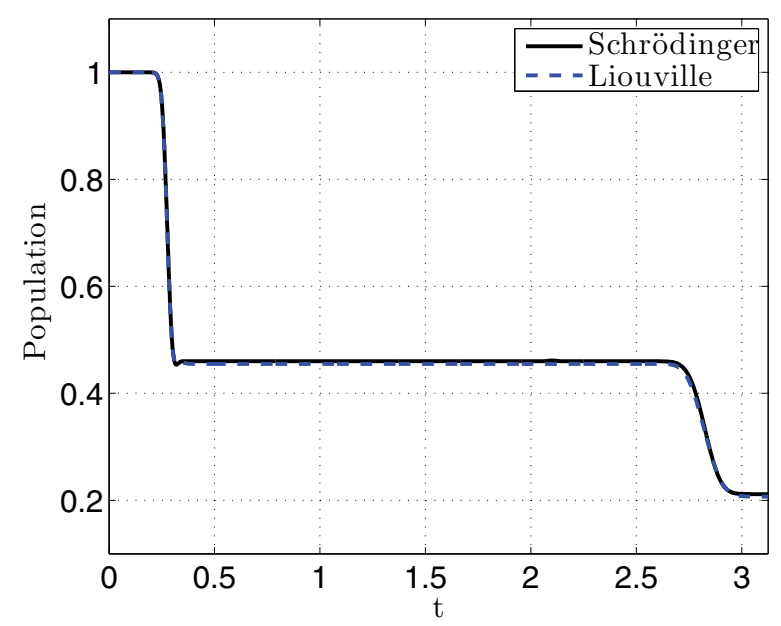

FIG. 2. Test 1: Time evolution of $P^{+}($see (4.4)).

We compare the results of our model with that of the Schrödinger. In Figure 3 we compare the results $\rho^{ \pm}$given by the two systems at time $t=0.75\left(A_{0}=2^{8}, a^{+}=1\right.$, $a^{-}=0, x_{0}=0.5$, and $p_{0}=-1$ ). In Figure 4 we check the evolution of the population on the first band $P^{+}$along the time.

Copyright $@$ by SIAM. Unauthorized reproduction of this article is prohibited. 


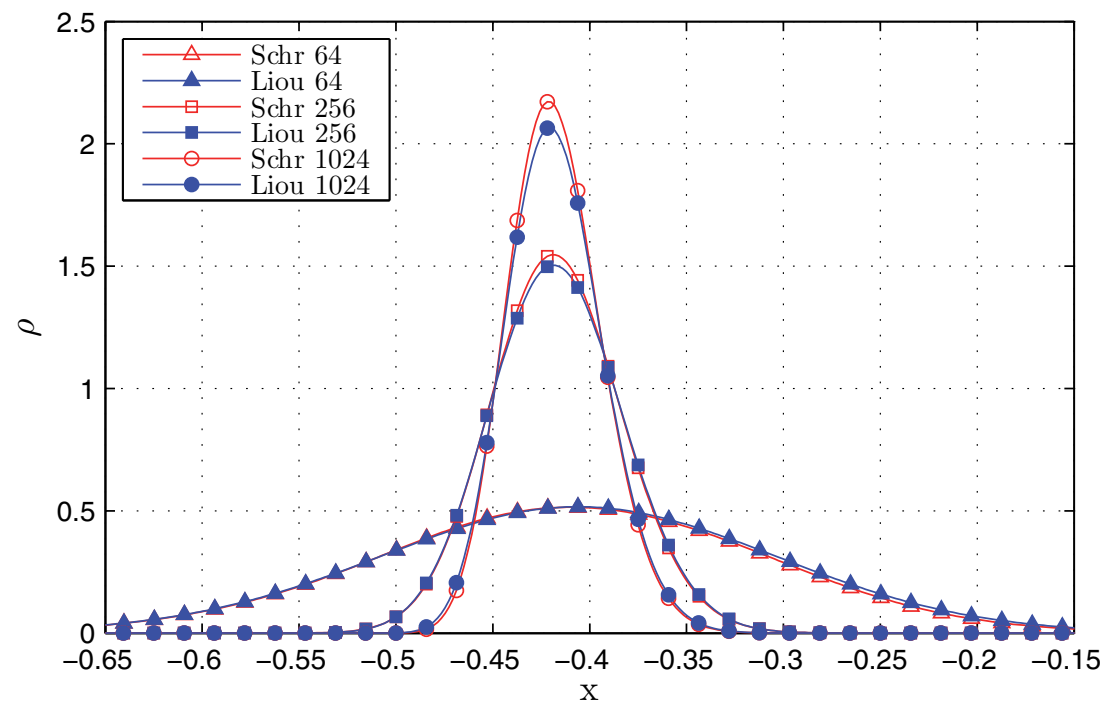

(a) $\rho_{\text {schr } / \text { liou }}^{+}$

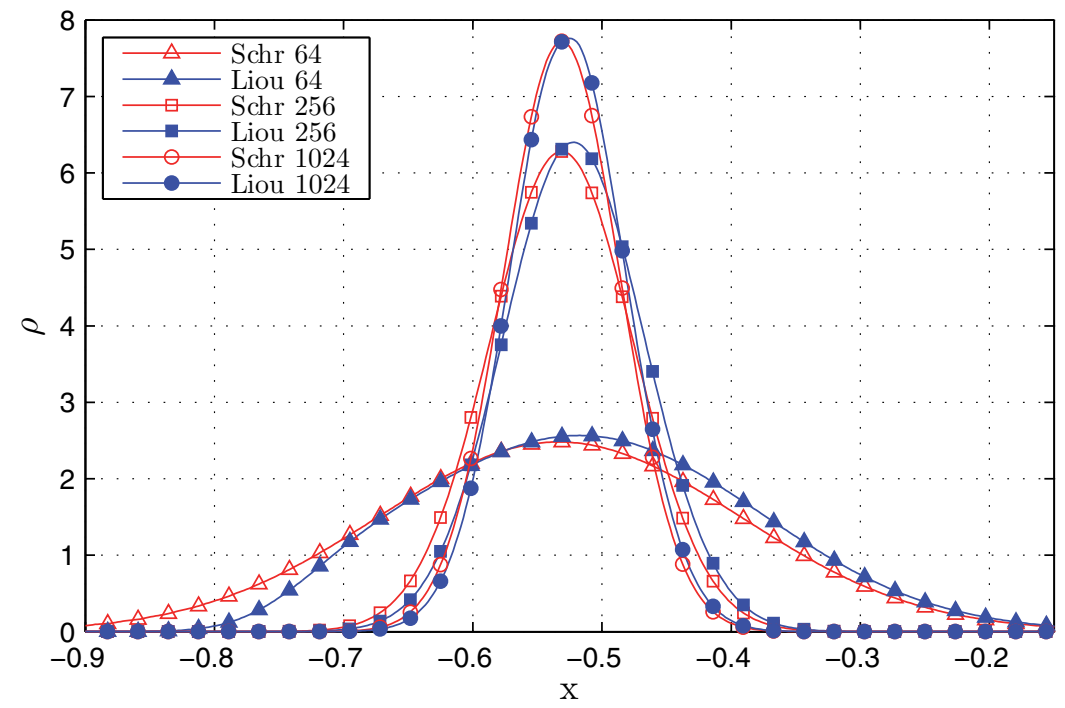

(b) $\rho_{\text {schr } / \text { liou }}^{-}$

FIG. 3. Test 1: The density functions $\rho_{\text {schr } / \text { liou }}^{ \pm}$for different $\varepsilon$ at time $t=0.75, \delta=\sqrt{\varepsilon} / 4$. The legend "Schr n" (or "Liou n") represents the solution of the Schrödinger equation (or the hybrid model) with $\varepsilon=1 / n$. Here, $A_{0}=2^{8}, a^{+}=1, a^{-}=0, x_{0}=0.5$, and $p_{0}=-1$.

Figure 5 shows that the hybrid model error (4.6) decreases at the rate of $O(\sqrt{\varepsilon})$ for $\delta=O(\sqrt{\varepsilon})$. The simulations show a good agreement between the two systems. In this figure, there are two lines, showing the different result with different choice of value of $C_{0}$, i.e., the width of the nonadiabatic region. One can see that there is not

Copyright (c) by SIAM. Unauthorized reproduction of this article is prohibited. 


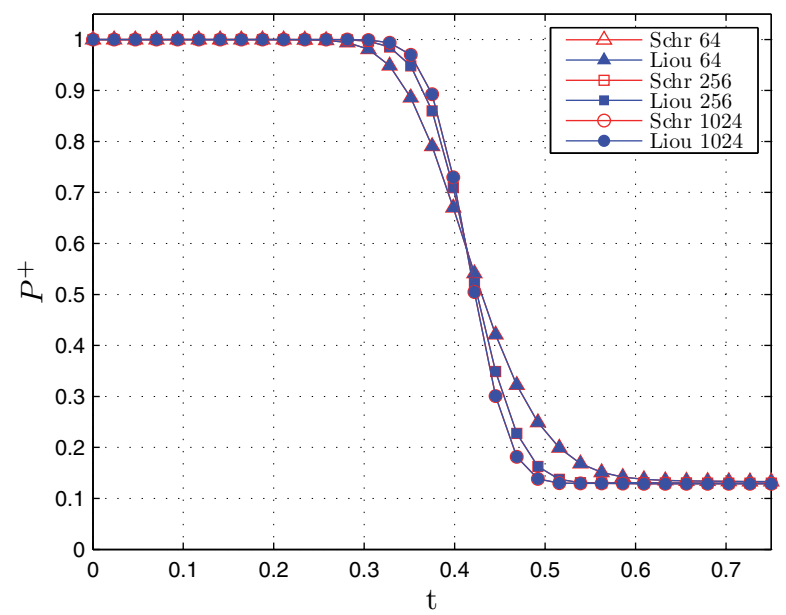

FIG. 4. Test 1: $1 D$ system simulation, time evolution of the population on the upper band $P_{\text {schr/liou }}^{+}$with $\delta=\sqrt{\varepsilon} / 4$. The legend "Schr $n$ " (or "Liou $n$ ") represents the solution of the Schrödinger equation (or the hybrid model) with $\varepsilon=1 / n$. Here, $A_{0}=2^{8}, a^{+}=1, a^{-}=0$, $x_{0}=0.5$, and $p_{0}=-1$.

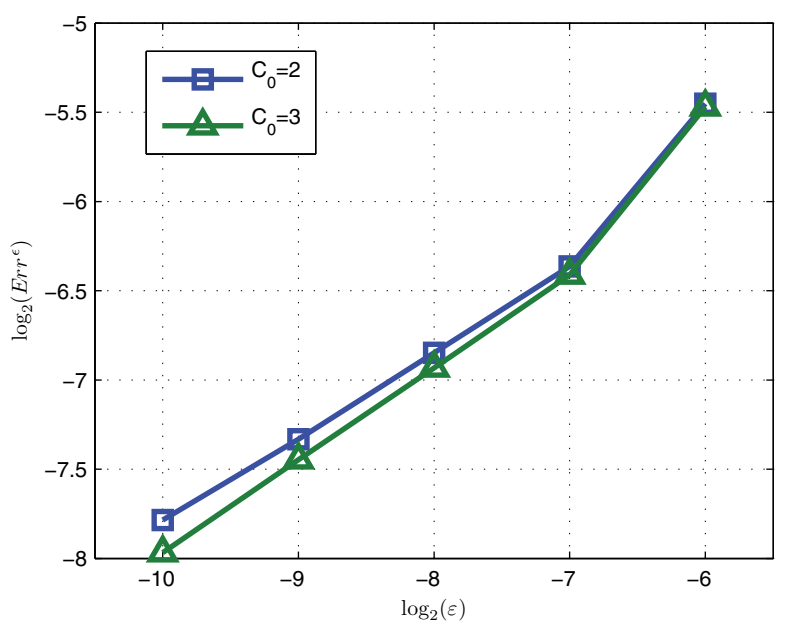

FIG. 5. Test 1: $1 D$ system simulation, $\operatorname{Err}^{\varepsilon}$ (4.6) decreases with a rate of $O(\sqrt{\varepsilon})$. Here, $A_{0}=2^{8}, a^{+}=1, a^{-}=0, x_{0}=0.5, p_{0}=-1$, and $t=0.75$.

too much difference for $C_{0}=2$ and $C_{0}=3$. So in the rest of the numerical examples, we take $C_{0}=3$.

We consider now a different initial condition. The initial wave packet for the Schrödinger equation is now given by (4.1)-(4.2) with $A_{0}=2^{8}, a^{+}=a^{-}=1 / \sqrt{2}$, $x_{0}=0.5$, and $p_{0}=-1$. This initial datum corresponds to a linear superposition of

Copyright (c) by SIAM. Unauthorized reproduction of this article is prohibited. 


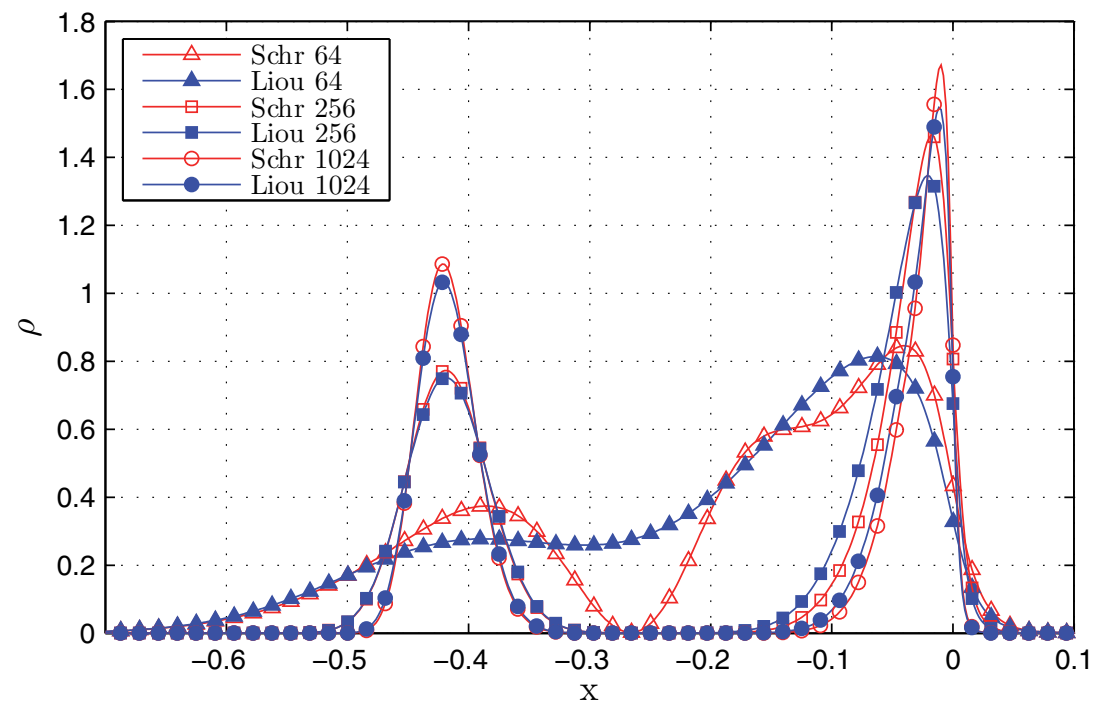

(a) $\rho_{\text {schr } / \text { liou }}^{+}$

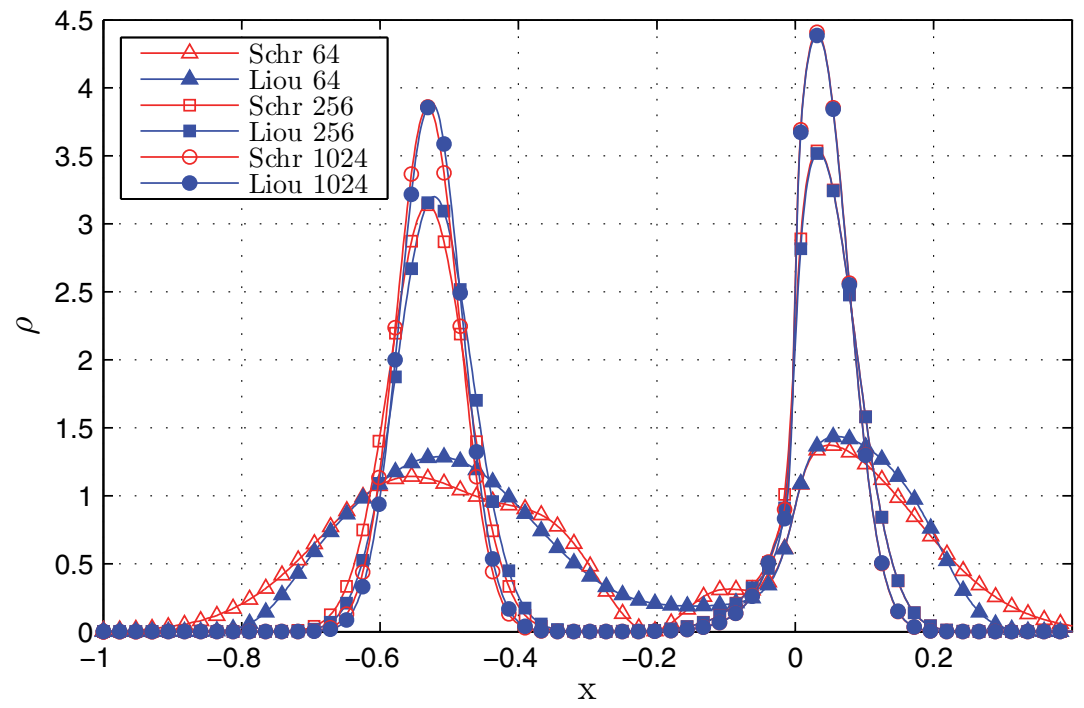

(b) $\rho_{\text {schr } / \text { liou }}^{-}$

FIG. 6. Test 1: $1 D$ system simulation, the density functions $\rho_{\text {schr/liou }}^{ \pm}$for different $\varepsilon$ at time $t=0.75, \delta=\sqrt{\varepsilon} / 4$. The legend "Schr $n$ " (or "Liou $n$ ") represents the solution of the Schrödinger equation (or the hybrid model) with $\varepsilon=1 / n$. Here, $A_{0}=2^{8}, a^{+}=a^{-}=1 / \sqrt{2}, x_{0}=0.5$, and $p_{0}=-1$.

two Gaussian packets that belong to the upper and lower bands, respectively.

In Figure 6 we compare the numerical results of $\rho^{ \pm}$to the Schrödinger equation and those to (2.18) at $t=0.75$. In Figure 7 , we show the evolution of the populations on the upper and lower bands $P^{ \pm}$with respect to time. We see that in the case of

Copyright (c) by SIAM. Unauthorized reproduction of this article is prohibited. 


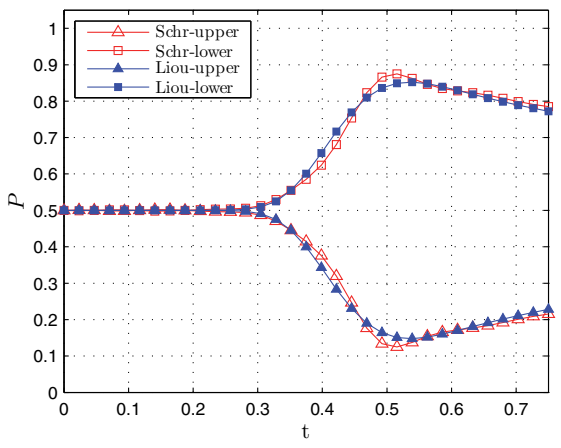

(a) $\varepsilon=2^{-6}$

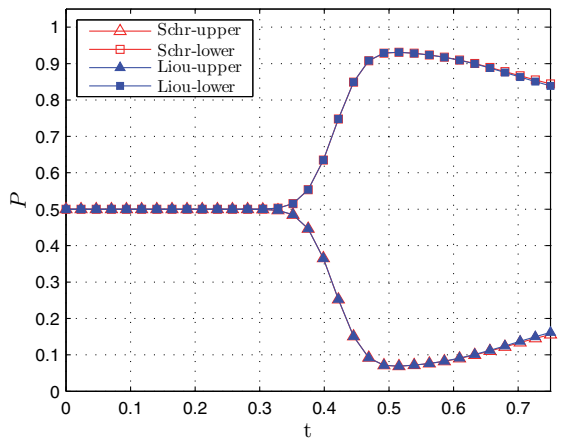

(c) $\varepsilon=2^{-8}$

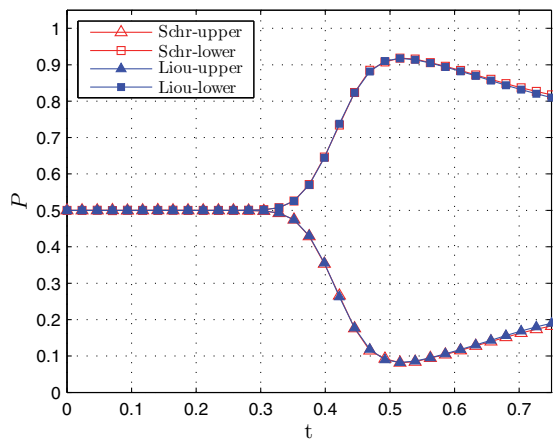

(b) $\varepsilon=2^{-7}$

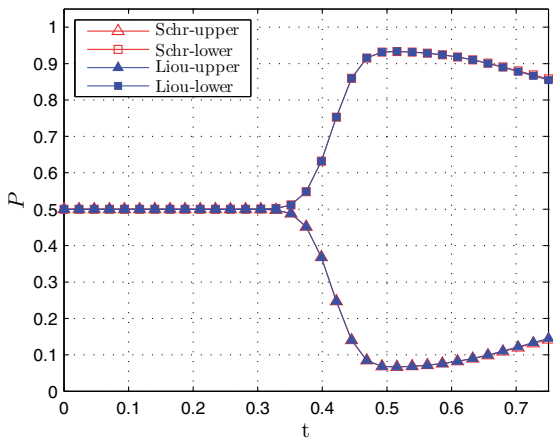

(d) $\varepsilon=2^{-9}$

FIG. 7. Test 1: $1 D$ system simulation, time evolution of the population on the upper and lower bands $P_{\text {schr/liou }}^{ \pm}(4.4) . \delta=\sqrt{\varepsilon} / 4$. The legend "Schr-upper" (or "Schr-lower") represents the population on upper (or lower) band given by the Schrödinger equation; "Liou-upper" (or "Lioulower") represents the population on upper (or lower) band given by the hybrid model. Here, $A_{0}=2^{8}$, $a^{+}=a^{-}=1 / \sqrt{2}, x_{0}=0.5$, and $p_{0}=-1$.

large $\varepsilon$ the semiclassical solution is not completely satisfactory. However, it is able to capture the main structure of the quantum interference between the upper and the lower band waves. For small $\varepsilon$, the two wave packets are well separated, and the solution of the hybrid model is in good agreement with the Schrödinger solution. Figure 8 shows that the cumulative error decreases at the rate of $O(\sqrt{\varepsilon})$.

4.2. Test 2: 1D system with concentrate initial data. In this subsection we repeat the numerical experiments in the previous subsection with the initial data changed to a Gaussian beam concentrate in both " $x$ " and " $p$ " by setting $A_{0}=1 / \varepsilon$.

We compare the results of our model with that of the Schrödinger. In Figure 9 we compare the results $\rho^{ \pm}$given by the two systems at time $t=0.75\left(A_{0}=1 / \varepsilon\right.$, $a^{+}=1, a^{-}=0, x_{0}=0.5$, and $p_{0}=-1$ ). In Figure 10 we check the evolution of the population on the first band $P^{+}$along the time. Figure 11 shows that the hybrid model error (4.6) decreases at the rate of $O(\sqrt{\varepsilon})$ for $\delta=O(\sqrt{\varepsilon})$. The simulations show a good agreement between the two systems. 


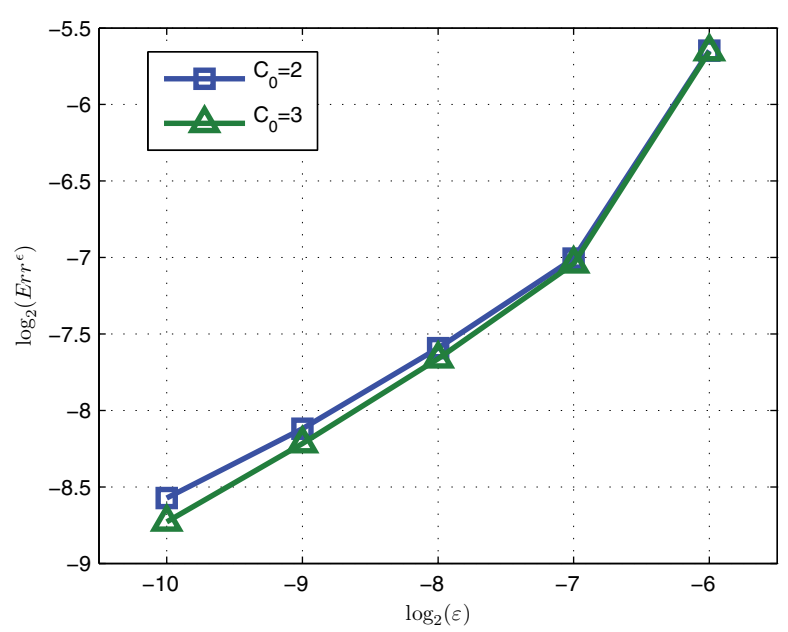

FIG. 8. Test 1: $1 D$ system simulation, $\operatorname{Err}^{\varepsilon}(4.6)$ as a function of $\varepsilon$ at $t=0.75$. Here, $A_{0}=2^{8}$, $a^{+}=a^{-}=1 / \sqrt{2}, x_{0}=0.5$, and $p_{0}=-1$.

We also study the system with the initial data distributed on both bands. The initial wave packet for the Schrödinger equation is now given by (4.1)-(4.2) with $A_{0}=1 / \varepsilon, a^{+}=a^{-}=1 / \sqrt{2}, x_{0}=0.5$, and $p_{0}=-1$. This initial datum corresponds to a linear superposition of two Gaussian packets that belong to the upper and lower bands, respectively.

In Figure 12 we compare the numerical results of our model (2.18) with the reference solution given by computing the Schrödinger equation directly at $t=0.75$. In Figure 13, we show the evolution of the populations on the upper and lower bands $P^{ \pm}$with respect to time. We see that in the case of large $\varepsilon$ the semiclassical solution is not completely satisfactory. However, it is able to capture the main structure of the quantum interference between the upper and the lower band waves. For small $\varepsilon$, the two wave packets are well separated, and the solution of the hybrid model is in good agreement with the Schrödinger solution. Figure 14 shows that the cumulative error decreases at the rate of $O(\sqrt{\varepsilon})$.

4.3. Test 3: $2 \mathrm{D}$ system. In this example, we deal with the problem in two dimensions with a pure state initial data. We set $u$ and $v$ as

$$
u(x)=x \quad \text { and } \quad v(x)=\sqrt{y^{2}+\delta^{2}} .
$$

We choose $\delta=\sqrt{\varepsilon} / 2$. The minimum of energy gap is $2 \delta$ and is located at the origin of the axis. The initial data for the Schrödinger equation are given in (4.1)-(4.2) with $a^{+}=1, a^{-}=0, x_{0}=5 \sqrt{\varepsilon}, y_{0}=0, p_{0 x}=-1$, and $p_{0 y}=0$. The Schrödinger equation is computed using the classical time-splitting spectral method, with $\Delta x=\Delta y=\varepsilon / 8$ 


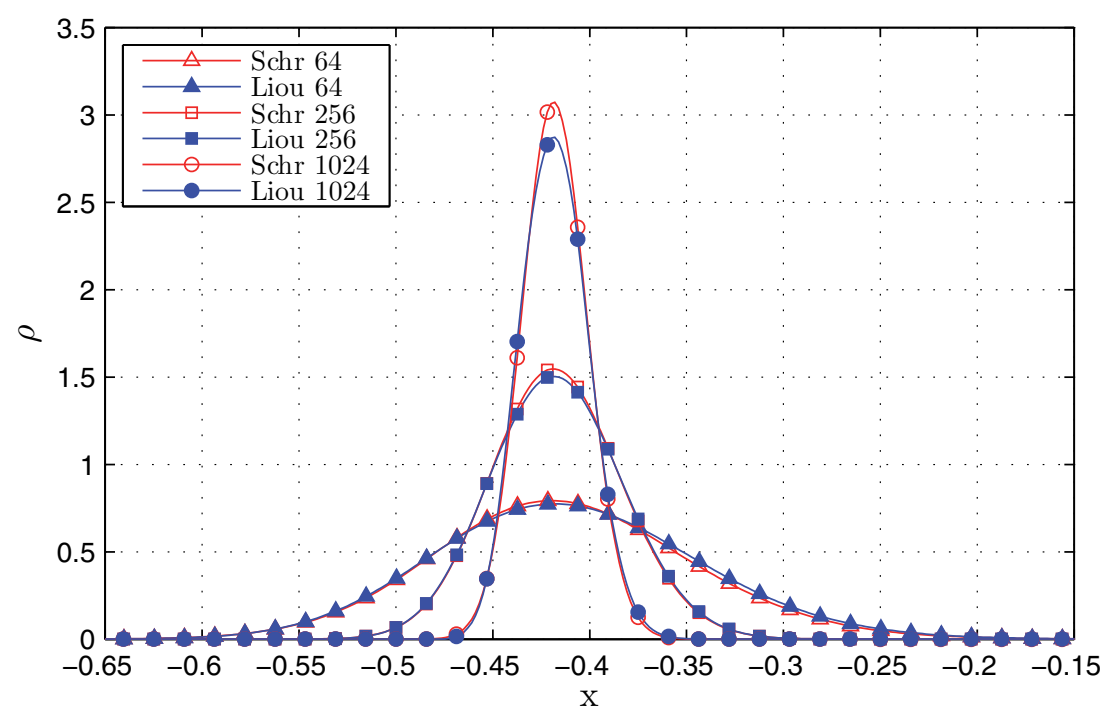

(a) $\rho_{\text {schr } / \text { liou }}^{+}$

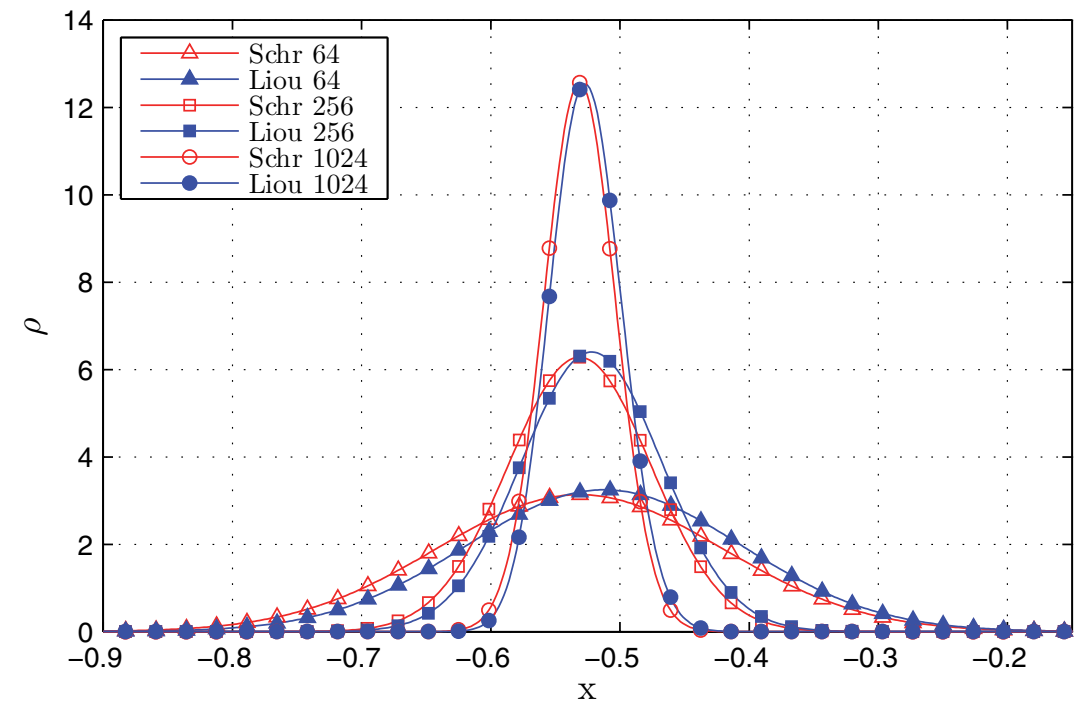

(b) $\rho_{\text {schr } / \text { liou }}^{-}$

FIG. 9. Test 2: Simulation with concentrate initial data. The density functions $\rho_{\text {schr } / \text { liou }}^{ \pm}$for different $\varepsilon$ at time $t=0.75, \delta=\sqrt{\varepsilon} / 4$. The legend "Schr $n$ " (or "Liou $n$ ") represents the solution of the Schrödinger equation (or the hybrid model) with $\varepsilon=1 / n$. Here, $A_{0}=1 / \varepsilon, a^{+}=1, a^{-}=0$, $x_{0}=0.5$, and $p_{0}=-1$.

and $\Delta t=5 \varepsilon^{\frac{3}{2}}$, and the hybrid model is computed with $\Delta x=\Delta p=h$ in the adiabatic regions and $\Delta x=\Delta p=h / 2$ in the nonadiabatic region, and $\Delta t=h / 8$, where $h=O(\sqrt{\varepsilon})$. In Figure 15 we show the snapshots of thedensity contour computed by

Copyright (c) by SIAM. Unauthorized reproduction of this article is prohibited. 


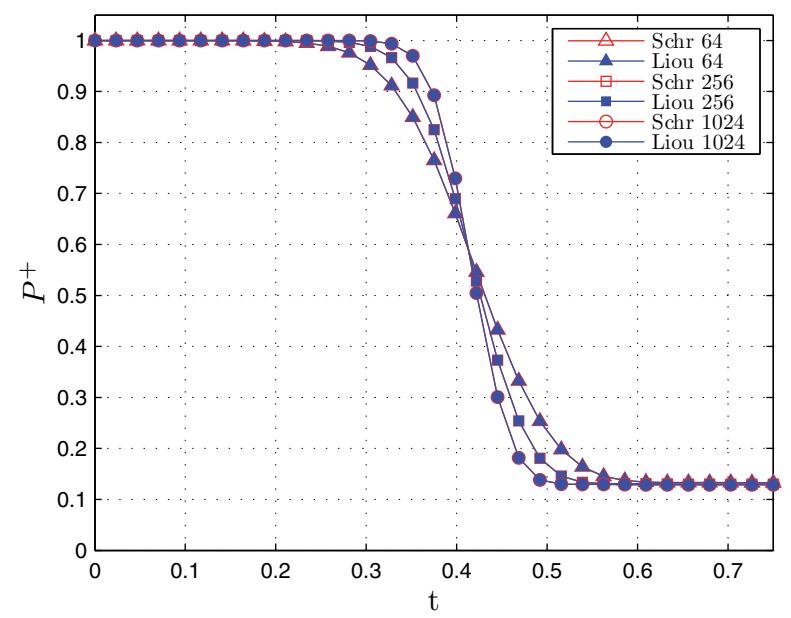

FIG. 10. Test 2: Simulation with concentrate initial data. Time evolution of the population on the upper band $P_{\text {schr } / \text { liou }}^{+}$with $\delta=\sqrt{\varepsilon} / 4$. The legend "Schr $n$ " (or "Liou n") represents the solution of the Schrödinger equation (or the hybrid model) with $\varepsilon=1 / n$. Here, $A_{0}=1 / \varepsilon, a^{+}=1$, $a^{-}=0, x_{0}=0.5$, and $p_{0}=-1$.

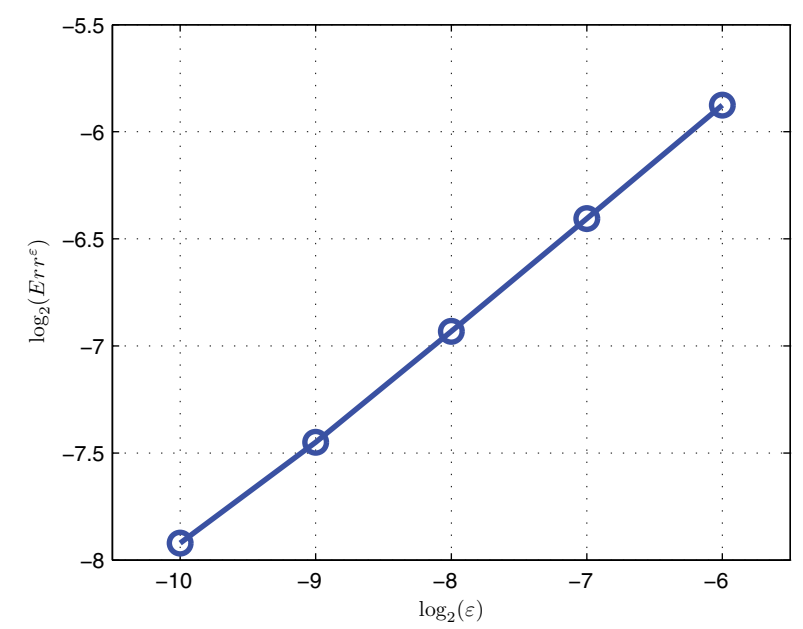

FIG. 11. Test 2: Simulation with concentrate initial data. $\operatorname{Err}^{\varepsilon}$ (4.6) decreases with a rate of $O(\sqrt{\varepsilon})$. Here, $A_{0}=1 / \varepsilon, a^{+}=1, a^{-}=0, x_{0}=0.5, p_{0}=-1$, and $t=0.75$.

the semiclassical model, while in Figure 16 we compare the evolution of the population on the first band $P^{+}$along the time given by the two systems. In Figure 15 we see that as time passes by, the density from the first band has some proportion jumping up to the second one. In Figure 16 we can clearly see that with resolved mesh the numerical solution to the semiclassical model agrees with that given by the Schrödinger equation.

Copyright $@$ ㅇ by SIAM. Unauthorized reproduction of this article is prohibited. 


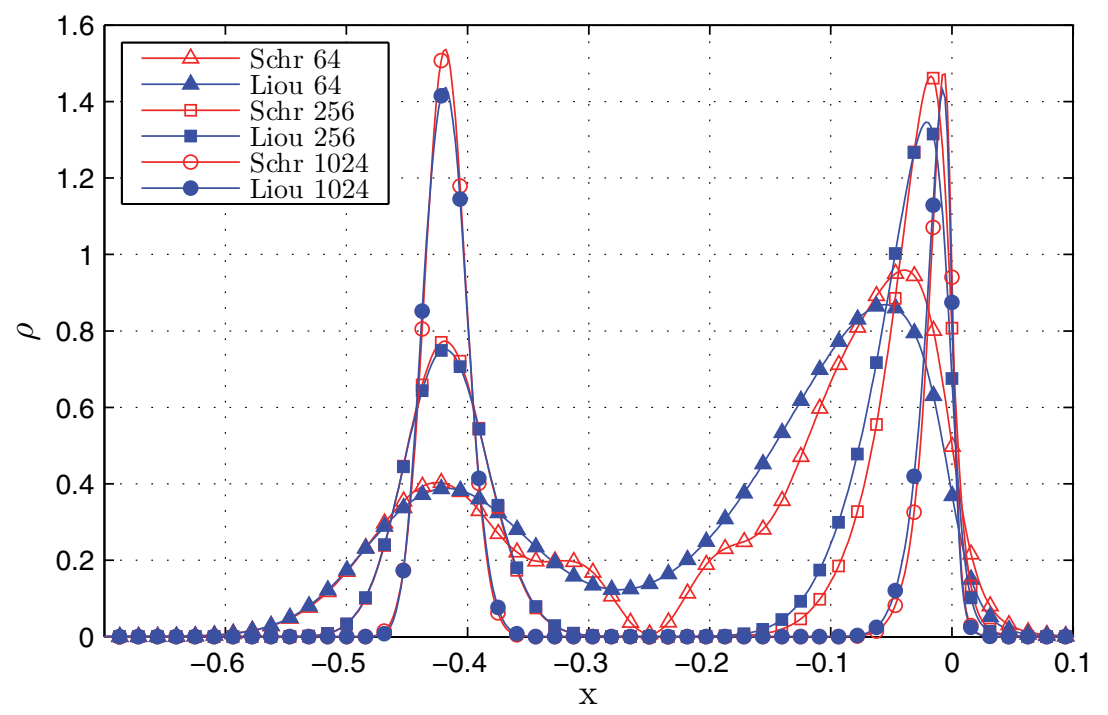

(a) $\rho_{\text {schr } / \text { liou }}^{+}$

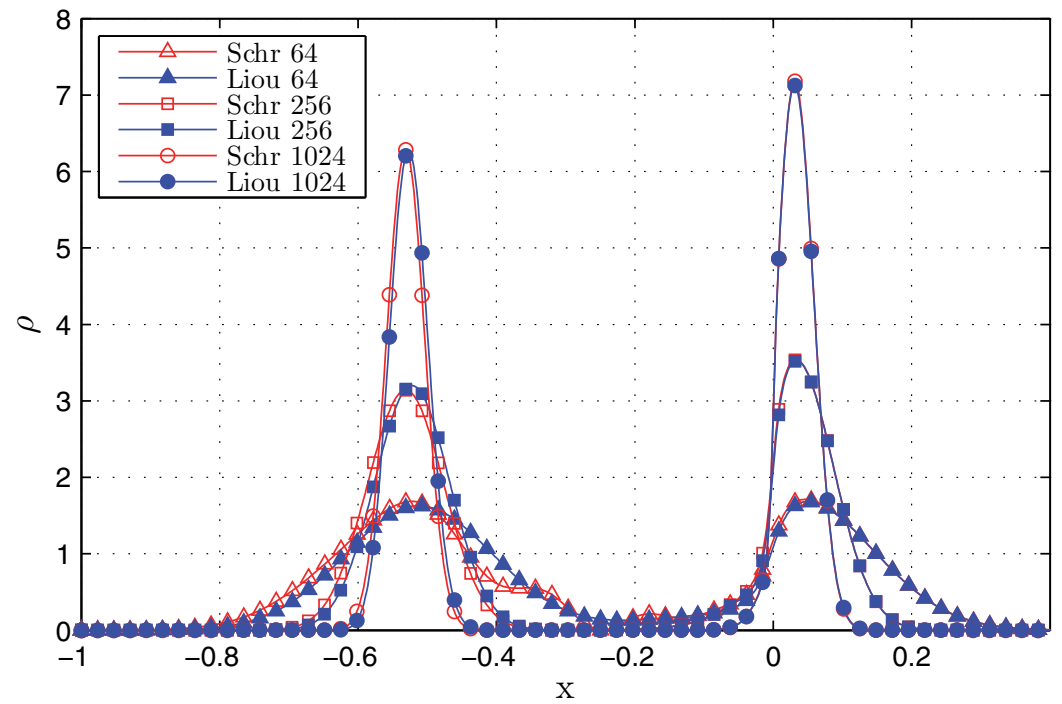

(b) $\rho_{\text {schr } / \text { liou }}^{-}$

FIG. 12. Test 2: Simulation with concentrate initial data. The density functions $\rho_{\text {schr } / \text { liou }}^{ \pm}$for different $\varepsilon$ at time $t=0.75, \delta=\sqrt{\varepsilon} / 4$. The legend "Schr $n$ " (or "Liou $n$ ") represents the solution of the Schrödinger equation (or the hybrid model) with $\varepsilon=1 / n$. Here, $A_{0}=1 / \varepsilon, a^{+}=a^{-}=1 / \sqrt{2}$, $x_{0}=0.5$, and $p_{0}=-1$.

Copyright $@$ by SIAM. Unauthorized reproduction of this article is prohibited. 


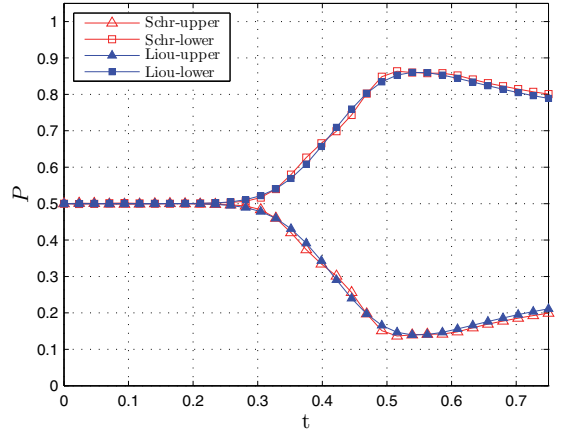

(a) $\varepsilon=2^{-6}$

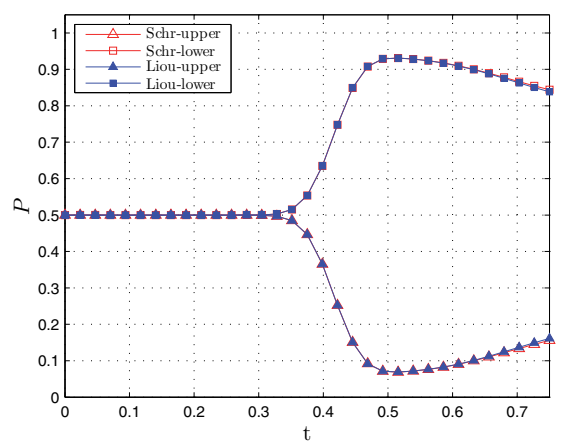

(c) $\varepsilon=2^{-8}$

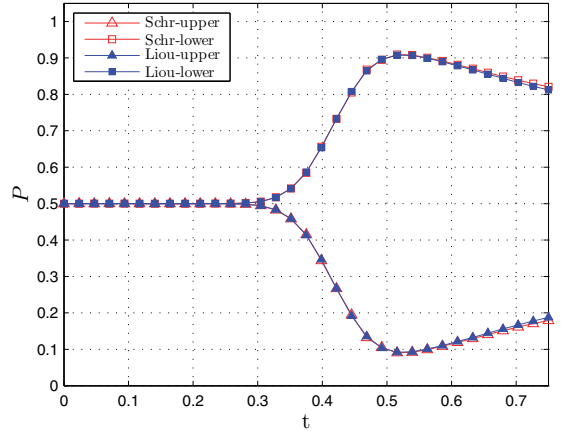

(b) $\varepsilon=2^{-7}$

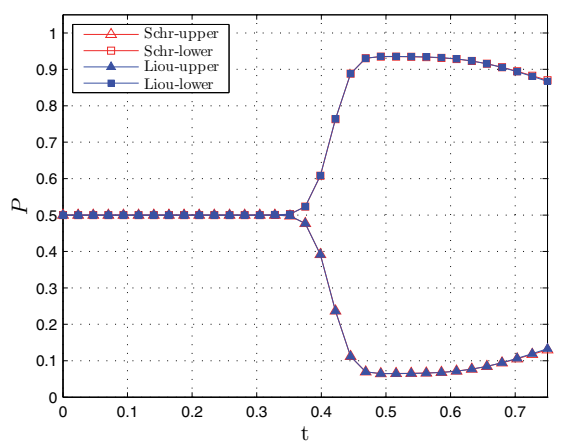

(d) $\varepsilon=2^{-9}$

FIG. 13. Test 2: Simulation with concentrate initial data. Time evolution of the population on the upper and lower bands $P_{\text {schr/liou }}^{ \pm}$(4.4). $\delta=\sqrt{\varepsilon} / 4$. The legend "Schr-upper" (or "Schr-lower") represents the population on upper (or lower) band given by the Schrödinger equation; "Liou-upper" (or "Liou-lower") represents the population on upper (or lower) band given by the hybrid model. Here, $A_{0}=1 / \varepsilon, a^{+}=a^{-}=1 / \sqrt{2}, x_{0}=0.5$, and $p_{0}=-1$.

Copyright (c) by SIAM. Unauthorized reproduction of this article is prohibited. 


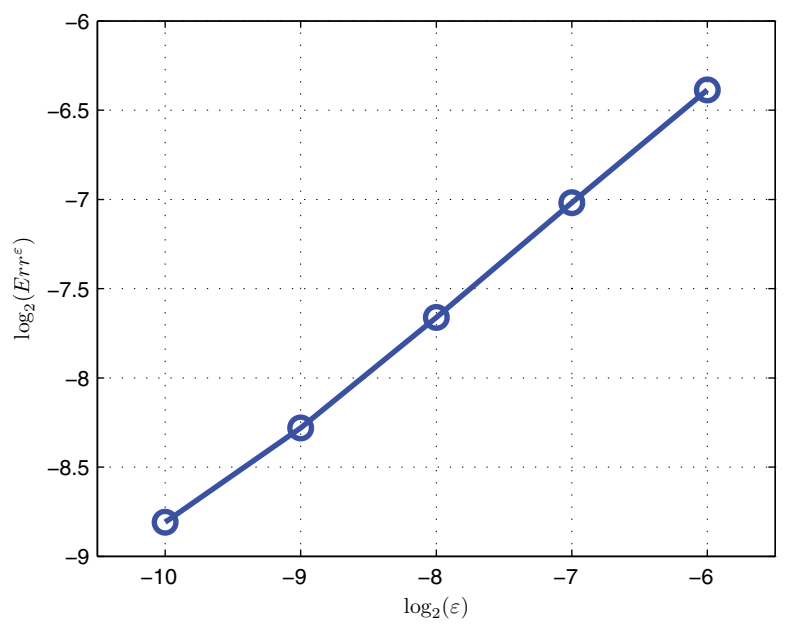

FIG. 14. Test 2: Simulation with concentrate initial data. $\operatorname{Err}^{\varepsilon}(4.6)$ as a function of $\varepsilon$ at $t=0.75$. Here, $A_{0}=1 / \varepsilon, a^{+}=a^{-}=1 / \sqrt{2}, x_{0}=0.5$, and $p_{0}=-1$.

Copyright $@$ by SIAM. Unauthorized reproduction of this article is prohibited. 

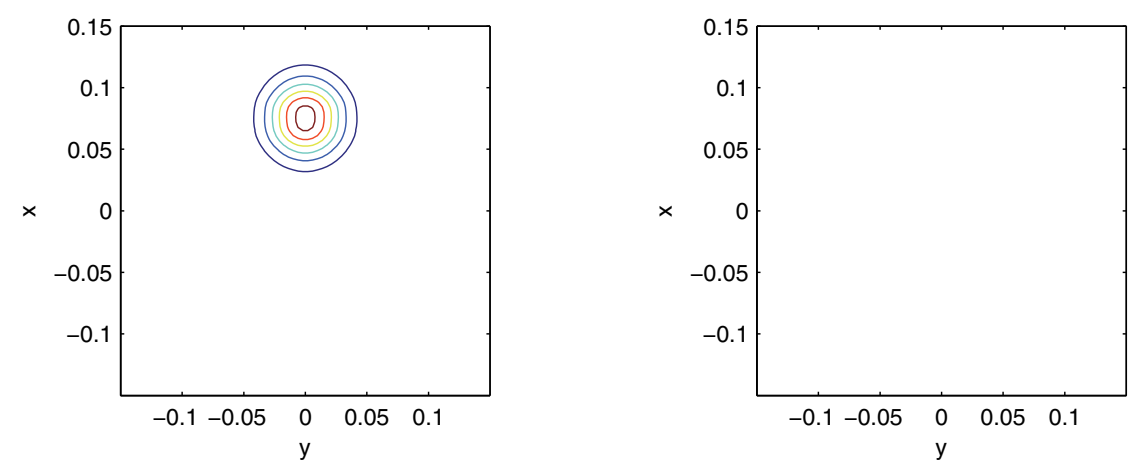

(a) $t=0.0781$
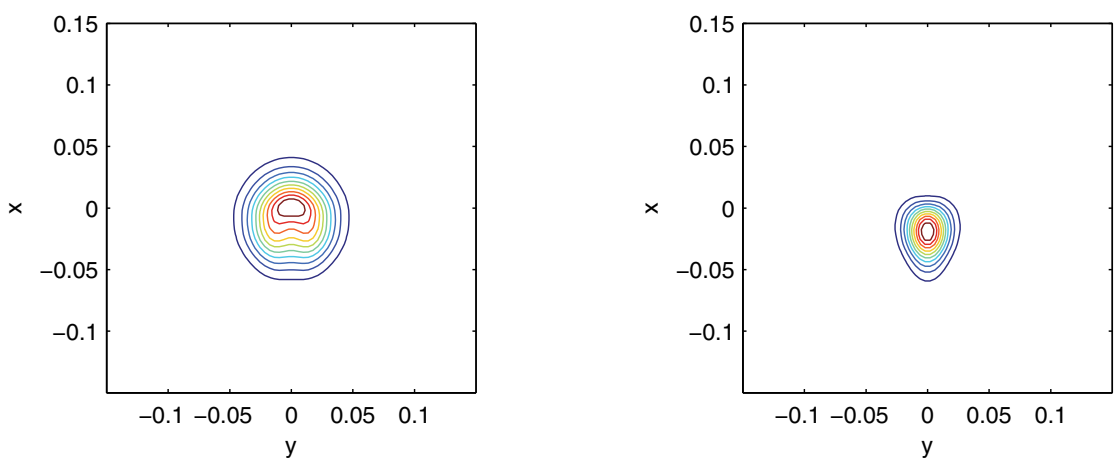

(b) $t=0.1562$
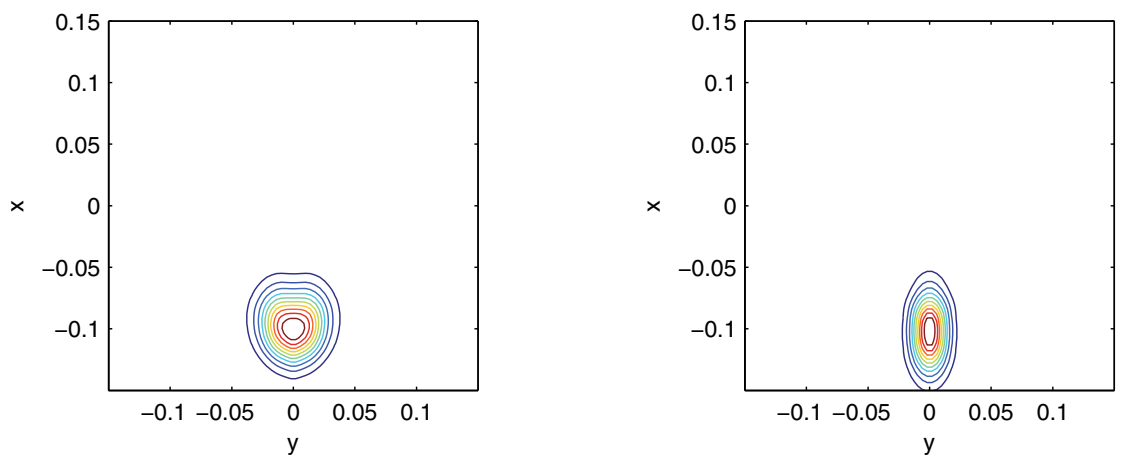

(c) $t=0.2344$

FIG. 15. Test 3: $1 D$ system simulation. The time evolution of density contour computed by the hybrid model. The left/right column are for $\rho_{\text {liou }}^{+} / \rho_{\text {liou }}^{-}$, the density on the upper/lower band (4.4). $\delta=\sqrt{\varepsilon} / 2$ and $\varepsilon=2^{-10}$. One can see in (b) around time $t=0.1562$ that the wave packet hits the crossing point and a portion of the mass jumps to the upper band.

Copyright $@$ by SIAM. Unauthorized reproduction of this article is prohibited. 


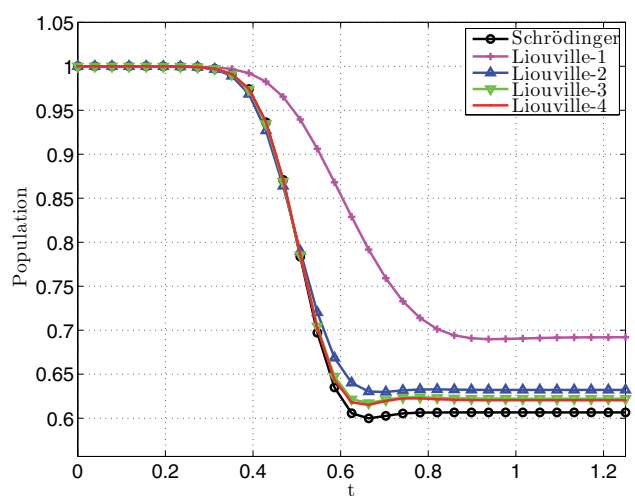

(a) $\varepsilon=2^{-6}$

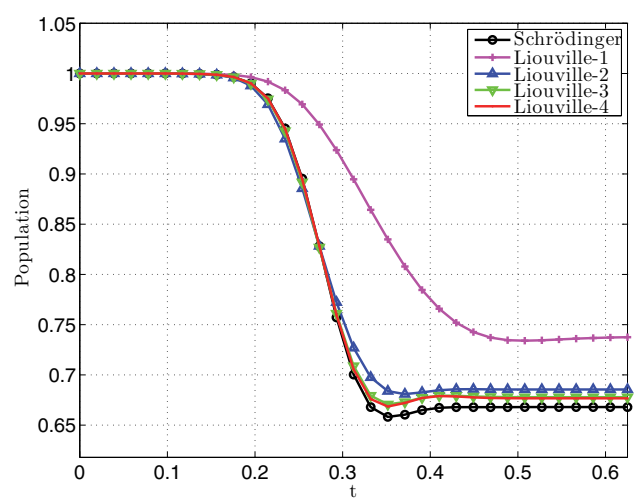

(c) $\varepsilon=2^{-8}$

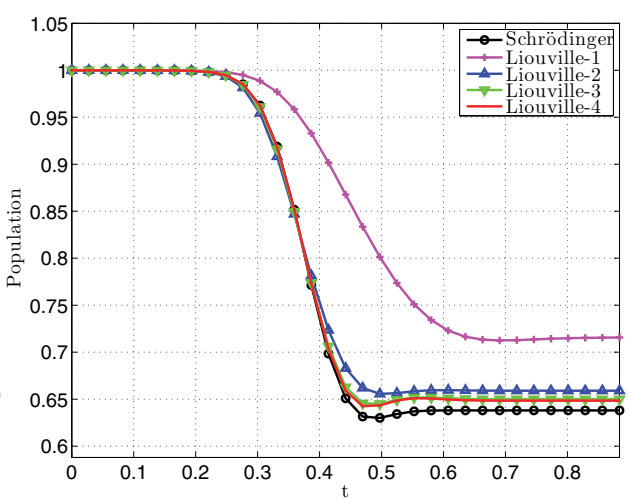

(b) $\varepsilon=2^{-7}$

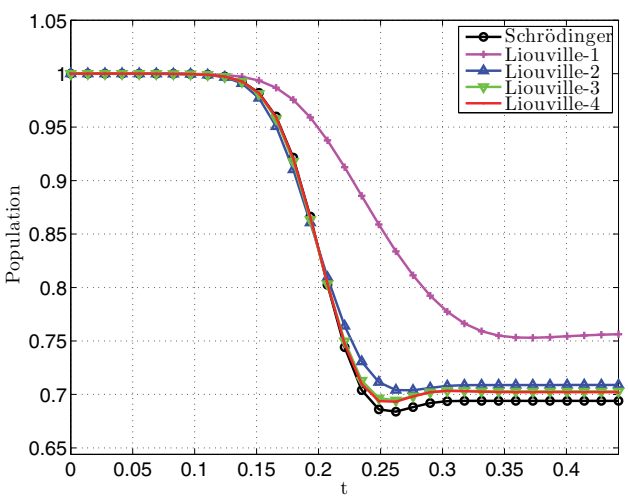

(d) $\varepsilon=2^{-9}$

FIG. 16. Test 3: 2D system simulation. Time evolution of the population on the upper band $P_{\text {schr/liou }}^{+}$(4.4). $\delta=\sqrt{\varepsilon} / 2$. The legend "Schrödinger" represents the solution of the Schrödinger equation, "Liouville- $j$ " represents the solution of the hybrid model with $\Delta x=\Delta p=h$ in the adiabatic regions and $\Delta x=\Delta p=h / 2$ in the nonadiabatic region, and $\Delta t=h / 8$, where $h=\sqrt{\varepsilon} / 2^{j-1}$ and $j=1,2,3,4$.

Copyright $@$ by SIAM. Unauthorized reproduction of this article is prohibited. 
5. Conclusion. In conclusion, we derived a semiclassical model for the nonadiabatic transition between different potential energy surfaces that goes beyond the classical Born-Oppenheimer approximation. By considering the complete Wigner matrix including the off-diagonal terms, our model is able to capture interesting physical phenomena such as the band-to-band transition, and the quantum correlation induced by the Berry connection. The hybrid model we proposed combines the classical adiabatic limit and the semiclassical model together to reduce the computational cost. The numerical simulations show that the hybrid model has a good agreement with the full quantum simulation.

Appendix. The derivation of the Hamiltonian $\boldsymbol{H}^{\prime}$. We give some details concerning the computation of the $H^{\prime}$ of (2.15). From the definition of $H^{\prime}$ we have

$$
\begin{aligned}
H^{\prime} & =\Theta(\boldsymbol{x}) \# H(\boldsymbol{x}, \boldsymbol{p}) \# \Theta^{\dagger}(\boldsymbol{x}) \\
& =\Theta(\boldsymbol{x}) \#(U(\boldsymbol{x}) \mathbb{I}+V(\boldsymbol{x})) \# \Theta^{\dagger}(\boldsymbol{x})+\Theta(\boldsymbol{x}) \#\left(\frac{\boldsymbol{p}^{2}}{2} \mathbb{I}\right) \# \Theta^{\dagger}(\boldsymbol{x}) \\
& =\operatorname{diag}\{U+E, U-E\}+\Theta(\boldsymbol{x}) \#\left(\frac{\boldsymbol{p}^{2}}{2} \mathbb{I}\right) \# \Theta^{\dagger}(\boldsymbol{x}) \\
& =\Lambda(\boldsymbol{x}, \boldsymbol{p})+\mathrm{i} \varepsilon \boldsymbol{p} \cdot \nabla_{\boldsymbol{x}} \Theta(\boldsymbol{x}) \Theta^{\dagger}(\boldsymbol{x})+\frac{\varepsilon^{2}}{2} \nabla_{\boldsymbol{x}} \Theta(\boldsymbol{x}) \cdot \nabla_{\boldsymbol{x}} \Theta^{\dagger}(\boldsymbol{x}),
\end{aligned}
$$

where $\Lambda=U(\boldsymbol{x})+\Lambda_{V}$ with $\Lambda_{V}$ defined in (1.7) and we used the first order expansion of the Moyal product

$$
\begin{aligned}
A \# B & =\sum_{n} \frac{1}{n !}\left(\frac{\mathrm{i} \varepsilon}{2}\right)^{n} A\left(\overleftarrow{\nabla_{\boldsymbol{x}}} \cdot \overrightarrow{\nabla_{\boldsymbol{p}}}-\overleftarrow{\nabla_{\boldsymbol{p}}} \cdot \overrightarrow{\nabla_{\boldsymbol{x}}}\right)^{n} B \\
& =A B+\frac{\mathrm{i} \varepsilon}{2}\left(\nabla_{\boldsymbol{x}} A \cdot \nabla_{\boldsymbol{p}} B-\nabla_{\boldsymbol{p}} A \cdot \nabla_{\boldsymbol{x}} B\right)+o(\varepsilon)
\end{aligned}
$$

In particular, we used

$$
\begin{aligned}
\Theta(\boldsymbol{x}) \#\left(\frac{\boldsymbol{p}^{2}}{2} \mathbb{I}\right) & =\frac{\boldsymbol{p}^{2}}{2} \Theta+\frac{\mathrm{i} \varepsilon}{2} \boldsymbol{p} \cdot \nabla_{\boldsymbol{x}} \Theta+\frac{1}{2}\left(\frac{\mathrm{i} \varepsilon}{2}\right)^{2} \Theta\left(\overleftarrow{\nabla_{\boldsymbol{x}}} \cdot \overrightarrow{\nabla_{\boldsymbol{p}}}\right)^{2}\left(\frac{\boldsymbol{p}^{2}}{2} \mathbb{I}\right)+o\left(\varepsilon^{2}\right) \\
& \left.=\frac{\boldsymbol{p}^{2}}{2} \Theta+\frac{\mathrm{i} \varepsilon}{2} \boldsymbol{p} \cdot \nabla_{\boldsymbol{x}} \Theta+\frac{1}{2}\left(\frac{\mathrm{i} \varepsilon}{2}\right)^{2} \Delta_{\boldsymbol{x}} \Theta+o\left(\varepsilon^{2}\right)\right]
\end{aligned}
$$

and

$$
\begin{aligned}
\left(\frac{\boldsymbol{p}^{2}}{2} \Theta(\boldsymbol{x})\right) \# \Theta^{\dagger}(\boldsymbol{x}) & =\frac{\boldsymbol{p}^{2}}{2}-\frac{\mathrm{i} \varepsilon}{2} \Theta\left(\boldsymbol{p} \cdot \nabla_{\boldsymbol{x}} \Theta^{\dagger}\right)+\frac{1}{2}\left(\frac{\mathrm{i} \varepsilon}{2}\right)^{2} \Theta \Delta_{\boldsymbol{x}} \Theta^{\dagger}, \\
\left(\boldsymbol{p} \cdot \nabla_{\boldsymbol{x}} \Theta(\boldsymbol{x})\right) \# \Theta^{\dagger}(\boldsymbol{x}) & =\boldsymbol{p} \cdot \nabla_{\boldsymbol{x}} \Theta \Theta^{\dagger}-\frac{\mathrm{i} \varepsilon}{2} \nabla_{\boldsymbol{x}} \Theta \cdot \nabla_{\boldsymbol{x}} \Theta^{\dagger}, \\
\Delta_{\boldsymbol{x}} \Theta(\boldsymbol{x}) \# \Theta^{\dagger}(\boldsymbol{x}) & =\Delta_{\boldsymbol{x}} \Theta \Theta^{\dagger}, \\
\Theta(\boldsymbol{x})\left(\boldsymbol{p} \cdot \nabla_{\boldsymbol{x}} \Theta^{\dagger}(\boldsymbol{x})\right) & =-\left(\boldsymbol{p} \cdot \nabla_{\boldsymbol{x}} \Theta\right) \Theta^{\dagger} \\
-2 \nabla_{\boldsymbol{x}} \Theta(\boldsymbol{x}) \cdot \nabla_{\boldsymbol{x}} \Theta^{\dagger}(\boldsymbol{x}) & =\Theta \Delta_{\boldsymbol{x}} \Theta^{\dagger}+\Delta_{\boldsymbol{x}} \Theta \Theta^{\dagger} .
\end{aligned}
$$

Copyright $@$ by SIAM. Unauthorized reproduction of this article is prohibited. 


\section{REFERENCES}

[1] J. E. Avron and A. Elgart, Adiabatic theorem without a gap condition: Two-level system coupled to quantized radiation field, Phys. Rev. A, 58 (1998), pp. 4300-4306.

[2] W. BaO, S. Jin, AND P. A. Markowich, On time-splitting spectral approximations for the Schrödinger equation in the semiclassical regime, J. Comput. Phys., 175 (2002), pp. 487524.

[3] D. R. BAtes, Collisions involving the crossing of potential energy curves, Proc. Roy. Soc. London Ser. A, 257 (1960), pp. 22-31.

[4] A. Böhm, A. Mostafazadeh, H. Koizumi, Q. Niu, and J. Zwanziger, The Geometric Phase in Quantum Systems: Foundations, Mathematical Concepts, and Applications in Molecular and Condensed Matter Physics, Springer-Verlag, Berlin, 2003.

[5] M. Born And R. Oppenheimer, Zur Quantentheorie der Molekeln [On the quantum theory of molecules], Ann. Phys., 389 (1927), pp. 457-484 (in German).

[6] R. Bourquin, V. Gradinaru, and G. A. Hagedorn, Nonadiabatic transitions near avoided crossings: Theory and numerics, J. Math. Chem., 50 (2011), pp. 602-619.

[7] L. Chai, S. Jin, And Q. Li, Semi-classical models for the Schrödinger equation with periodic potentials and band crossings, Kinet. Relat. Models, 6 (2013), pp. 505-532.

[8] C. Fermanian-Kammerer and C. Lasser, Wigner measures and codimension two crossings, J. Math. Phys., 44 (2003), pp. 507-527.

[9] C. Fermanian-Kammerer and C. Lasser, Single switch surface hopping for molecular quantum dynamics, J. Math. Chem., 50 (2011), pp. 620-635.

[10] P. Gérard, P. A. Markowich, N. J. Mauser, and F. Poupaud, Homogenization limits and Wigner transforms, Comm. Pure Appl. Math., 50 (1997), pp. 323-379.

[11] L. Gosse And P. A. Markowich, Multiphase semiclassical approximation of an electron in a one-dimensional crystalline lattice, I, J. Comput. Phys., 197 (2004), pp. 387-417.

[12] G. A. Hagedorn, A time dependent Born-Oppenheimer approximation, Comm. Math. Phys., 77 (1980), pp. 1-19.

[13] G. A. Hagedorn, High order corrections to the time-dependent Born-Oppenheimer approximation I: Smooth potentials, Ann. of Math. (2), 124 (1986), pp. pp. 571-590.

[14] G. A. Hagedorn, High order corrections to the time-dependent Born-Oppenheimer approximation. II: Coulomb systems, Comm. Math. Phys., 117 (1988), pp. 387-403.

[15] G. A. Hagedorn, Proof of the Landau-Zener formula in an adiabatic limit with small eigenvalue gaps, Comm. Math. Phys., 136 (1991), pp. 433-449.

[16] G. A. Hagedorn, Classification and normal forms for avoided crossings of quantummechanical energy levels, J. Phys. A, 31 (1998), pp. 369-383.

[17] G. A. Hagedorn And A. Joye, Landau-Zener transitions through small electronic eigenvalue gaps in the Born-Oppenheimer approximation, Ann. Inst. H. Poincaré Phys. Théor., 68 (1998), p. $85-134$.

[18] G. A. Hagedorn And A. Joye, Molecular propagation through small avoided crossings of electron energy levels, Rev. Math. Phys., 11 (1999), pp. 41-101.

[19] G. A. Hagedorn and A. Joye, A time-dependent Born-Oppenheimer approximation with exponentially small error estimates, Comm. Math. Phys., 223 (2001), pp. 583-626.

[20] G. A. Hagedorn and A. Joye, Mathematical analysis of Born-Oppenheimer approximations, in Spectral Theory and Mathematical Physics, F. Gesztesy, P. Deift, C. Galvez, P. Perry, and W. Schlag, eds., AMS, Providence, RI, 2007, pp. 203-226.

[21] M. F. Herman, Generalization of the geometric optical series approach for nonadiabatic scattering problems, J. Chem. Phys., 76 (1982), pp. 2949-2958.

[22] L. Hörmander, The Weyl calculus of pseudo-differential operators, Comm. Pure Appl. Math., 32 (1979), pp. 359-443.

[23] V. JaKŠIĆ And J. Segert, On the Landau-Zener formula for two-level systems, J. Math. Phys., 34 (1993), pp. 2807-2820.

[24] S. Jin AND P. QI, A hybrid Schrödinger/Gaussian beam solver for quantum barriers and surface hopping, Kinet. Relat. Models, 4 (2011), pp. 1097-1120.

[25] S. Jin, P. QI, AND Z. ZHANG, An Eulerian surface hopping method for the Schrödinger equation with conical crossings, Multiscale Model. Simul., 9 (2011), pp. 258-281.

[26] S. Jin AND D. YIN, Computational high frequency waves through curved interfaces via the Liouville equation and geometric theory of diffraction, J. Comput. Phys., 227 (2008), pp. 61066139.

[27] A. Joye, H. Kunz, And C.-E. Pfister, Exponential decay and geometric aspect of transition probabilities in the adiabatic limit, Ann. Physics, 208 (1991), pp. 299-332.

Copyright $@$ by SIAM. Unauthorized reproduction of this article is prohibited. 
[28] A. Joye And C.-E. Pfister, Exponentially small adiabatic invariant for the Schrödinger equation, Comm. Math. Phys., 140 (1991), pp. 15-41.

[29] R. Kapral and G. Ciccotti, Mixed quantum-classical dynamics, J. Chem. Phys., 110 (1999), pp. 8919-8929.

[30] T. KAтo, On the adiabatic theorem of quantum mechanics, J. Phys. Soc. Japan, 5 (1950), pp. 435-439.

[31] C. Lasser, T. Swart, and S. Teufel, Construction and validation of a rigorous surface hopping algorithm for conical crossings, Commun. Math. Sci., 5 (2007), pp. 789-814.

[32] R. J. LeVeque, Numerical Methods for Conservation Laws, 2nd ed., Birkhäuser Verlag, Basel 1992.

[33] O. Morandi, Multiband Wigner-function formalism applied to the Zener band transition in a semiconductor, Phys. Rev. B, 80 (2009), 024301.

[34] O. Morandi, Effective classical Liouville-like evolution equation for the quantum phase-space dynamics, J. Phys. A, 43 (2010), 365302.

[35] O. Morandi And F. SchürRer, Wigner model for Klein tunneling in graphene, Commun. Appl. Ind. Math., 2 (2011), e-360.

[36] O. Morandi And F. SchürRer, Wigner model for quantum transport in graphene, J. Phys. A, 44 (2011), pp. 265-301.

[37] G. Nenciu And V. Sordoni, Semiclassical limit for multistate Klein-Gordon systems: Almost invariant subspaces, and scattering theory, J. Math. Phys., 45 (2004), pp. 3676-3696.

[38] G. Panati, H. Spohn, and S. Teufel, Space-adiabatic perturbation theory in quantum dynamics, Phys. Rev. Lett., 88 (2002), pp. 205405-205408.

[39] T. Paul and P.-L. Lions, Sur les mesures de Wigner, Rev. Mat. Iberoamericana, 9 (1993), pp. 553-618.

[40] V. Rousse, Landau-Zener transitions for eigenvalue avoided crossings in the adiabatic and Born-Oppenheimer approximations, Asymptot. Anal., 37 (2004), pp. 293-328.

[41] H. Spohn and S. Teufel, Adiabatic decoupling and time-dependent Born-Oppenheimer theory, Comm. Math. Phys., 224 (2001), pp. 113-132.

[42] S. Teufel, Adiabatic Perturbation Theory in Quantum Dynamics, Springer-Verlag, Berlin, 2003.

[43] J. C. Tully, Trajectory surface hopping approach to nonadiabatic molecular collisions: The reaction of $\mathrm{H}^{+}$with $\mathrm{D}_{2}$, J. Chem. Phys., 55 (1971), pp. 562-572.

[44] J. C. Tully, Molecular dynamics with electronic transitions, J. Chem. Phys., 93 (1990), pp. 1061-1071.

[45] J. von Neumann And E. P. Wigner, Über merkwürdige diskrete Eigenwerte, in The Collected Works of E. P. Wigner, A. S. Wightman, ed., Vol. A/1, Springer, Berlin, Heidelberg, 1993, pp. 291-293.

[46] H. WeYL, Quantenmechanik und Gruppentheorie, Z. Phys., 46 (1927), pp. 1-46.

[47] G. A. Worth and L. S. Cederbaum, Beyond Born-Oppenhermer: Molecular dynamics through a conical intersection, Annu. Rev. Phys. Chem., 55 (2004), pp. 127-158.

[48] D. Xiao, M.-C. Chang, And Q. Niu, Berry phase effects on electronic properties, Rev. Modern Phys., 82 (2010), pp. 1959-2007.

[49] D. R. YARKonY, Nonadiabatic quantum chemistry-Past, present, and future, Chem. Rev., 112 (2012), pp. 481-498.

[50] C. Zener, Non-adiabatic crossing of energy levels, Proc. R. Soc. Lond. A, 137 (1932), pp. 696702 .

Copyright (c) by SIAM. Unauthorized reproduction of this article is prohibited. 